Amnesia in Logopenic Progressive Aphasia

\title{
Understanding the neural basis of episodic amnesia in logopenic progressive aphasia: a multimodal neuroimaging study
}

Siddharth Ramanan ${ }^{\mathrm{a}, \mathrm{b}, \mathrm{c}}$, Lars Marstaller ${ }^{\mathrm{d}}$, John R. Hodges ${ }^{\mathrm{a}, \mathrm{b}, \mathrm{e}}$, Olivier Piguet ${ }^{\mathrm{a}, \mathrm{b}, \mathrm{c}}$, Muireann Irish ${ }^{\mathrm{a}, \mathrm{b}, \mathrm{c} *}$

a The University of Sydney, Brain and Mind Centre, Sydney, New South Wales, Australia

b The University of Sydney, School of Psychology, Sydney, New South Wales, Australia

c ARC Centre of Excellence in Cognition and its Disorders, Sydney, New South Wales, Australia

d Department of Psychology, Swansea University, Swansea, Wales, United Kingdom

e The University of Sydney, School of Medical Sciences, Sydney, New South Wales, Australia

*Corresponding author: Associate Professor Muireann Irish, Brain and Mind Centre, The University of Sydney, 100 Mallett Street, Camperdown, NSW 2050, Sydney, Australia; Tel: +61 29114 4165; e-mail: muireann.irish@sydney.edu.au

Word count (abstract): 290 words

Word count (manuscript): 6,293 words

Number of tables: 3

Number of figures: 5 
Amnesia in Logopenic Progressive Aphasia

Total number of display items: 8

Supplementary material: 1 supplementary file

Number of tables (supplementary): 8

Number of figures (supplementary): 2 
Amnesia in Logopenic Progressive Aphasia

\begin{abstract}
Logopenic progressive aphasia (LPA) is a neurodegenerative disorder characterised by profound naming and sentence repetition disturbances, attributable to disproportionately left-sided temporo-parietal atrophy. Accumulating evidence suggests, in addition to language impairments, the presence of stark verbal and nonverbal episodic memory impairments in LPA. The neurocognitive bases of such impairments, however, remain to be clarified. Here, we characterised episodic memory disruption and its corresponding grey and white matter correlates in the LPA syndrome.
\end{abstract} Nineteen LPA patients were contrasted with 23 matched typical Alzheimer's disease (AD) patients and 31 healthy Controls on standardized verbal and nonverbal episodic delayed recall measures. Participants further underwent structural magnetic resonance and diffusion-weighted imaging. Significant verbal memory deficits were evident in both patient groups, with LPA patients performing at an intermediate level to AD and Controls. For nonverbal memory, however, LPA performance was indistinguishable from that of $\mathrm{AD}$, with both groups displaying marked impairments relative to Controls. Whole-brain voxel-based morphometry analyses revealed significant left temporoparietal and left hippocampal atrophy in the LPA group. Covariate analyses showed that verbal and nonverbal amnesia in LPA correlated with grey matter integrity of bilateral frontoparietal and left medial temporal lobe regions. Notably, the common regions underpinning verbal and nonverbal memory dysfunction in LPA were the left orbitofrontal cortex and bilateral angular gyri in the inferior parietal cortex. The bilateral angular gyri, along with prefrontal and hippocampal regions further emerged as disease-general correlates of verbal and nonverbal memory performance. Alterations in mean diffusivity in structural connections between the left angular gyrus and medial temporal lobes were further associated with verbal memory performance in all 
Amnesia in Logopenic Progressive Aphasia

participants. Our findings reveal, for the first time, the presence of pervasive memory impairments in LPA mediated by degeneration of a distributed prefrontal-hippocampalparietal network, and disrupted parieto-hippocampal structural connectivity.

Keywords: Primary Progressive Aphasia; Alzheimer's disease; episodic memory; angular gyrus; hippocampus 
Amnesia in Logopenic Progressive Aphasia

\section{INTRODUCTION}

Logopenic progressive aphasia (LPA) is a rare clinical syndrome whose prototypical features include markedly reduced spontaneous speech in the context of phonological errors, word-finding, sentence repetition, and sentence comprehension difficulties (Gorno-Tempini et al., 2008; Gorno-Tempini et al., 2011). Semantic comprehension and grammatical processing abilities, by contrast, are relatively spared in early stages of the syndrome (Gorno-Tempini et al., 2008; Gorno-Tempini et al., 2004). The unique clinical profile of LPA is attributable to a breakdown in multiple cognitive processes such as verbal working memory, lexical retrieval, and phonological processing, which collectively support spontaneous speech, naming, and repetition abilities (Henry \& Gorno-Tempini, 2010; Leyton, Piguet, Savage, Burrell, \& Hodges, 2012). On the neuroanatomical level, the locus of atrophy in LPA is predominantly centred on the left inferior parietal cortex, left lateral temporal and posterior perisylvian cortical regions (around the left inferior/middle/superior temporal gyri) (Gorno-Tempini et al., 2008; Krishnan et al., 2017; Leyton et al., 2012; Rohrer et al., 2010; Teichmann et al., 2013), further affecting underlying white matter connectivity between these regions (Galantucci et al., 2011). By contrast, evidence for early involvement of the medial temporal lobes (MTL) and hippocampi in LPA is mixed, with some studies pointing to their relative integrity (Teichmann et al., 2013; Win et al., 2017), while others demonstrate early degradation of left-lateralized MTL/hippocampal regions (GornoTempini et al., 2004; Rohrer et al., 2013). Pathologically, the majority of LPA patients harbour abnormal levels of cortical $\beta$-amyloid burden, pathognomic of Alzheimer's disease (AD), at levels which are comparable to those seen in individuals with the typical amnestic presentation of AD (Chare et al., 2014; Grossman, 2010; Leyton et al., 2011; Rohrer, Rossor, \& Warren, 2012). Together, this has led to the 
Amnesia in Logopenic Progressive Aphasia

reconceptualization of LPA as an atypical variant of $\mathrm{AD}$, presenting predominantly with language dysfunction (Ahmed, de Jager, Haigh, \& Garrard, 2012).

Based on these underlying patterns of neurodegeneration, a common assumption is that LPA is primarily a disorder of language, while the cardinal feature of typical AD is that of episodic memory dysfunction. Counter to this view, however, is the observation of significant episodic memory impairments early in the LPA disease trajectory, across verbal (Piguet, Leyton, Gleeson, Hoon, \& Hodges, 2015; Win et al., 2017) and nonverbal (Piguet et al., 2015; Ramanan et al., 2016) delayed recall measures. Moreover, the severity of episodic amnesia in LPA is comparable to that observed in typical AD, particularly when nonverbal episodic delayed recall measures are employed (Ramanan et al., 2016). These objective memory deficits in LPA are complemented by subjective patient-reports (Magnin et al., 2013) and carer-reports (Ramanan et al., 2016) of everyday memory difficulties. Based on this emerging evidence, we suggest that in parallel with canonical language disturbances, LPA can also be conceptualised as an amnesic syndrome.

The observation of verbal and nonverbal episodic memory impairments in LPA raises important questions regarding the candidate neurocognitive mechanisms driving these deficits. From a cognitive standpoint, it is reasonable to propose that episodic memory deficits in LPA arise largely due to gross lexical processing impairments characteristic of this syndrome. A recent study supports this proposal by demonstrating an association between verbal episodic delayed recall impairments and lexical retrieval performance in LPA, attributable to a common neural substrate centred on the left middle temporal gyrus (Win et al., 2017). The left middle temporal gyrus is well established as a lexical 
processing hub in the perisylvian language network (reviewed by Gow, 2012) and appears particularly vulnerable in early stages of LPA (Gorno-Tempini et al., 2008). While the lexical retrieval deficit offers a parsimonious account of verbal episodic memory dysfunction in LPA, it does not accommodate findings of non-verbal memory impairments on tasks which circumvent lexical retrieval demands (e.g., Butts et al., 2015; Piguet et al., 2015; Ramanan et al., 2016). As such, the core mechanisms underlying episodic memory dysfunction in LPA remain unclear.

The objectives of the present study were twofold. First, we aimed to characterise episodic memory performance across verbal and non-verbal domains in LPA compared to typical $\mathrm{AD}$ patients, matched across multiple demographic, clinical, and cognitive variables. In line with recent reports (Piguet et al., 2015; Ramanan et al., 2016), we predicted verbal and nonverbal episodic memory impairments in LPA relative to healthy Controls, comparable to that observed in typical AD. Second, we sought to establish the underlying grey and white matter correlates of episodic memory dysfunction in LPA and AD, employing an a priori hypothesis-driven approach based on the canonical profiles of neural degeneration in each syndrome. We were particularly interested in a modulating role of the left inferior parietal cortex in episodic memory dysfunction in LPA, given (i) its strong structural and functional connections with the MTL memory network (reviewed by Ramanan, Piguet, \& Irish, 2018), and (ii) early disruption of its white matter connectivity with the MTL in LPA (Gorno-Tempini et al., 2008; Tu, Leyton, Hodges, Piguet, \& Hornberger, 2016). Using convergent grey and white matter neuroimaging approaches, the current study represents the first formal characterisation of the neural underpinnings of episodic memory dysfunction in LPA. 
Amnesia in Logopenic Progressive Aphasia

\section{MATERIALS AND METHODS}

We report how we determined our sample size, all data exclusions, all inclusion/exclusion criteria, whether inclusion/exclusion criteria were established prior to data analysis, all manipulations, and all measures in the study.

\subsection{Participants}

A total of 73 participants were recruited through FRONTIER, the frontotemporal dementia research group at the Brain and Mind Centre, the University of Sydney, Australia. Nineteen patients with a clinical diagnosis of LPA (Gorno-Tempini et al., 2011) who presented with early anomia, and difficulties with word-finding and sentence repetition were included. Twenty-three patients with a clinical diagnosis of probable $\mathrm{AD}$ with predominantly amnestic presentation were included (McKhann et al., 2011). Atypical variants of AD such as Posterior Cortical Atrophy or dysexecutive variants of $\mathrm{AD}$ were excluded.

Diagnoses were established by consensus among a multidisciplinary team comprising a senior neurologist (J.R.H.), a clinical neuropsychologist, and an occupational therapist based on comprehensive clinical and neuropsychological assessment, and structural neuroimaging. Disease severity was established using the Clinical Dementia Rating Frontotemporal Lobar Degeneration Sum of Boxes score (CDR-FTLD SoB; Knopman et al., 2008). In addition, the CDR-FTLD Memory subdomain score was used as a metric of clinician-rated memory impairment in patients. Carers completed the Cambridge Behavioural Inventory - Revised (CBI-R; Wear et al., 2008) as an index of behavioural changes in the patient. 
Amnesia in Logopenic Progressive Aphasia

Thirty-one healthy control participants were selected through a research volunteer panel and local community clubs and were matched to patient groups for age, sex, and education. All controls scored 88 or above on the Addenbrooke's Cognitive Examination - Revised (ACE-R: Mioshi, Dawson, Mitchell, Arnold, \& Hodges, 2006) - a global index of cognitive functioning that assesses orientation, memory, verbal fluency, language, and visuospatial processing. All controls scored 0 on the CDRFTLD SoB measure. Exclusion criteria for all participants included history of cerebrovascular disease, significant head injury, drug and alcohol abuse, other primary neurological, psychiatric, or mood disorders, and limited English proficiency.

All participants or their Person Responsible provided written informed consent in accordance with the Declaration of Helsinki. This study was approved by the South Eastern Sydney Local Health District and the University of New South Wales ethics committees.

\subsection{General neuropsychological assessment}

Participants underwent a comprehensive neuropsychological assessment including tests of language, executive function, and memory. Overall cognitive functioning was measured using the ACE-R total score (Mioshi et al., 2006). The language subscale of the ACE-R provided a global impression of language performance across single word and sentence comprehension and repetition, reading, writing, and naming subtests. Targeted assessments of naming, single word repetition and word comprehension from the Sydney Language Battery (SYDBAT: Savage et al., 2013) were further administered. Each of the SYDBAT subtests have a maximum score of 30 with demonstrated sensitivity to language impairments in Primary Progressive Aphasia 
Amnesia in Logopenic Progressive Aphasia

subtypes (Savage et al., 2013). Executive dysfunction was assessed using the time difference between parts B and A of the Trail Making Test (TMT B-A: Reitan, 1958). Forward and backward digit span tests were used as indices of auditory attention and working memory, respectively (Wechsler, 1997). Verbal letter fluency (F, A, S) tests were used to measure word generation and controlled word retrieval abilities (Strauss, Sherman, \& Spreen, 2006). Finally, visuo-constructional abilities were assessed using the Copy score from the Rey-Osterrieth Complex Figure test (ROCF: Osterrieth, 1944).

\subsection{Episodic memory assessments}

Episodic memory was assessed across verbal and nonverbal domains in keeping with previous studies (Irish, Piguet, Hodges, \& Hornberger, 2014; Ramanan et al., 2016). First, overall memory performance was indexed using the memory subcomponent from the ACE-R (max score $=26$ ), which comprises immediate recall, retrograde memory, and learning, delayed recall and recognition of a Name and Address (see Irish et al., 2016).

Verbal episodic memory was assessed using the Rey Auditory Verbal Learning Test (RAVLT: Schmidt, 1996). Participants learn 15 words over five consecutive acquisition trials $(\max$ score on each trial $=15)$ followed by a filled 30 -minute delay, after which delayed recall and recognition are assessed (max score for each $=15)$. The main score of interest here was the delayed recall score $(\max$ score $=15$, hereafter, referred to as verbal delayed recall score). For visualization purposes, this score was expressed as a percentage $[($ raw score / max score $) * 100]$. 
Amnesia in Logopenic Progressive Aphasia

Nonverbal episodic memory was assessed using the delayed recall component of the ROCF test (Osterrieth, 1944). Here, participants are required to copy a complex figure comprising multiple elements (Copy trial: $\max$ score $=36$ ) and must reproduce the figure from memory following a delay of 3 minutes ( $\max$ score for delayed recall $=36$ ). To control for executive and visuo-constructional demands, a percentage retained score was calculated [(delayed recall score / copy score $) * 100$, hereafter, referred to as nonverbal delayed recall score] and this score formed the main score of interest for nonverbal memory. The recognition component of the ROCF test was not administered in this study.

\subsection{Image acquisition}

Seventy-one participants (18 LPA, 23 AD, 30 Controls) underwent a structural MRI of the brain using a 3T Philips MRI scanner with standard quadrature head coil (eight channels). Whole-brain $\mathrm{T}_{1}$-weighted images were acquired using the following sequences: coronal acquisition, matrix $256 \times 256 \mathrm{~mm}, 200$ slices, voxel size $=1 \mathrm{~mm}^{3}$, echo time/repetition $=2.6 / 5.8 \mathrm{~ms}$, flip angle $\alpha=8^{\circ}$. Fifty-eight participants additionally underwent diffusion-weighted MRI. Two sets of whole-brain echo planar images were acquired with 32 non-collinear gradient directions, matrix 96 x 96 mm, 55 slices, voxel size $=2.5 \mathrm{~mm}^{3}$, repetition time/echo time/inversion time: $8400 / 68 / 90 \mathrm{~ms}$, b-value $=$ $1000 \mathrm{~s} / \mathrm{mm}^{2}$, field of view $=240 \times 240 \mathrm{~mm}$.

\subsection{VBM analyses}

Voxel-based morphometry (VBM) analyses were conducted using FSL (FMRIB Software Library: https://fsl.fmrib.ox.ac.uk/fsl/fslwiki) according to a standardized preprocessing pipeline involving brain extraction (Smith, 2002), tissue segmentation 
(Zhang, Brady, \& Smith, 2001), and non-linear registration methods (Andersson, Jenkinson, \& Smith, 2007a, 2007b) to align brain-extracted images to the Montreal Neurological Institute (MNI) standard space. Following image pre-processing, wholebrain general linear models were employed to investigate grey matter intensity differences between groups (corrected for Family-Wise Error at $p<.005$ ). Correlations were then performed to investigate relationships between grey matter intensity and episodic memory performance (corrected for False Discovery Rate at $p<.05$ ). Finally, inclusive masking analyses were used to determine common neural correlates of episodic memory within (i.e., disease-specific) and between (i.e., disease-general) patient groups. Full details of image pre-processing and VBM analyses are provided in Supplementary Methods.

It should be noted that all VBM correlation analyses were conducted combining each patient group with the Control group (i.e., LPA and Controls, AD and Controls), allowing increased statistical power in detecting disease-specific associations. An important concern to address in this regard is whether group distributions due to an underlying bimodal distribution (i.e., different modes for patients vs. Controls) explain emergent correlation findings. To ensure that an underlying bimodal distribution did not drive the correlation results, we first combined each patient group with the Control group and inspected the modes for both verbal and nonverbal delayed recall performance (in line with similar methods by Bertoux et al., 2014). Importantly, across these contrasts, a single mode that was different from the combined group's mean verbal and nonverbal delayed recall score underlay the distribution (see Supplementary Table 1 for details). These findings were further corroborated by visual inspection of histogram distributions, which revealed the presence of a single, distinct peak 
Amnesia in Logopenic Progressive Aphasia

suggesting a unimodal distribution underlying each combined patient-Control group performance on both verbal and nonverbal memory measures. Together, this suggests that the emergent findings in the VBM correlation analyses are not attributable to the presence of underlying group differences between patient and Control test performance.

\subsection{Diffusion Tensor Imaging analyses}

Diffusion weighted imaging data were available for 58 participants (15 LPA, 19 AD, 24 Controls). Briefly, Tract-Based Spatial Statistics (TBSS: Smith et al., 2006) were run in FSL to perform a skeleton-based analysis of white matter fractional anisotropy (FA). Diffusion weighted images for each participant were corrected for eddy-currents and co-registered using non-linear registration (FNIRT: Andersson et al., 2007a, 2007b) to MNI standard space using their respective 3D $\mathrm{T}_{1}$-weighted structural $\mathrm{MR}$ image. Following this, tensor models were fit to diffusion weighted images, and FA maps for participants were generated, which were averaged to produce a group mean FA image. General linear models were employed to examine white matter intensity differences between groups (corrected for Family-Wise Error at $p<.005$ ). Full details of image pre-processing and Diffusion Tensor Imaging analyses are provided in Supplementary Methods.

\subsection{Probabilistic tractography}

To complement the TBSS analyses, we further sought to delineate specific white matter microstructural changes in the structural connections between the left angular gyrus (AG) in the inferior parietal cortex and the left MTL/hippocampus. The rationale for examining structural connectivity between these particular regions was based on i) causal evidence demonstrating critical roles for both regions in episodic memory 
retrieval (see Berryhill, 2012; Nadel \& Moscovitch, 1997; Ramanan \& Bellana, 2019; Ramanan et al., 2018), ii) the emergence of both left AG and left hippocampus in our VBM atrophy and correlation analyses for the LPA group, iii) prior knowledge of tract anatomy (Catani, Howard, Pajevic, \& Jones, 2002) demonstrating structural connectivity between the left AG (particularly, the ventral AG or area PGp; Caspers et al., 2011) and the left MTL (particularly, the parahippocampal gyrus/posterior hippocampus) via the inferior longitudinal fasciculus (ILF) (Caspers et al., 2011; Rushworth, Behrens, \& Johansen-Berg, 2006; Uddin et al., 2010), and iv) prior evidence for early disruption of structural integrity of the left ILF in LPA (Tu et al., 2016).

For probabilistic tractography analyses, seed and target region, along with waypoint and exclusion masks were first defined in MNI space. The seed region mask comprised the left ventral AG, defined from the Jülich histological atlas implemented in FSL. The target region mask comprised the left posterior hippocampus. This was further used as a 'waypoint' mask, instructing the algorithm to only retain streamlines that passed through this point. Finally, a right-hemisphere mask and a bilateral frontal lobe mask were employed as exclusion masks. All masks were linearly transformed from MNI space to each subject's native space for probabilistic tractography.

Probabilistic tractography was initiated from all voxels within the diffusion space seed mask while considering fibre pathway restrictions posed by the waypoint and exclusion masks. The resultant fibre tract was normalised, thresholded and the following indices of overall microstructural integrity of the white matter connectivity were computed:

i. $\quad$ Fractional anisotropy (FA) 
Amnesia in Logopenic Progressive Aphasia

ii. Mean diffusivity (MD) - indicating average molecular diffusion along the three eigenvectors of the tensor, with higher values indicating a higher mean diffusivity.

The extracted tractmetric values for all participants were subsequently used to examine group differences. Finally, associations between tractmetric values and verbal and nonverbal episodic delayed recall measures were examined. Full details of probabilistic tractography analyses are provided in Supplementary Methods.

\subsection{Tract-of-no-interest approach}

To validate the probabilistic tractography correlational analyses, we further employed a 'tract-of-no-interest' approach using a control white matter tract hypothesized to not play a substantive role in episodic delayed recall. For this, a mask was created for the left corticospinal tract (running caudally from the precentral gyrus to the cerebral peduncle) based on the Johns Hopkins University white matter tractography atlas, integrated into FSLview (Hua et al., 2008). Similar to the above analyses, FA and MD values for this tract were extracted and correlated with episodic memory performance.

\subsection{Statistical analyses}

Data were analysed using RStudio v3.3.0. For all behavioural data, Shapiro-Wilk tests were first used to assess normality of distributions. For normally-distributed continuous variables (e.g., age of disease onset), group differences were examined using $t$-tests and ANOVAs, with Sidak correction for post-hoc comparisons for ANOVA outputs. Wilcoxon-Mann-Whitney tests were used to examine group differences on nonnormally distributed continuous variables (e.g., disease severity). For categorical variables (e.g., sex), Chi-square tests were used. Group differences on episodic memory 
Amnesia in Logopenic Progressive Aphasia

performance were examined using ANOVA with Sidak correction for post-hoc comparisons. The alpha level to determine statistical significance was set at $p<.05$ or below. Effect sizes for all ANOVA statistics are denoted using partial eta-squared values $\left(\eta_{p}^{2}\right)$, while Cohen's $d$ values denote effect sizes of post-hoc LPA-AD comparisons. All $F$-statistics, $p$-values, and $\eta_{p}^{2}$ values are indicated in the respective tables. Two-tailed Pearson's correlations were administered to examine associations between verbal/nonverbal episodic memory and performance on neuropsychological tests separately for LPA and AD groups. Due to the large number of correlations undertaken, all $p$-values for correlation analyses were corrected for multiple comparisons using Benjamini-Hochberg (false-discovery rate) method to control for Type-I error (Benjamini \& Hochberg, 1995).

For statistical analysis of probabilistic fibre tracking metrics, group differences were assessed using ANOVAs with Sidak post-hoc corrections. Associations between episodic delayed recall measures and tract metrics were examined using two-tailed Pearson's correlation coefficients, employing a conservative alpha of $p \leq .01$ to control for Type-I error. Given the relatively small sample sizes for the probabilistic tractography analyses for both LPA $(N=15)$ and $\mathrm{AD}(N=19)$ groups, correlations were computed combining each patient group with the Control group (i.e., LPA and Controls, $\mathrm{AD}$ and Controls) to increase statistical power. Importantly, there was no evidence for the presence of a bimodal distribution underpinning each combined patient-Control group performance on both verbal and nonverbal delayed recall performance (Supplementary Table 1), suggesting that the findings emerging from the correlation analyses could not simply be attributed to group differences. 
Amnesia in Logopenic Progressive Aphasia

\subsection{Data availability}

The ethical requirement to ensure patient confidentiality precludes public archiving of our data. Researchers who would like to access the raw data should contact the corresponding author who will liaise with the ethics committee that approved the study, and accordingly, as much data that is required to reproduce the results will be released to the individual researcher. The code used for this project has been made available for review on the Open Science Framework website (https://osf.io/c5azm/). No part of the study procedures or analyses were preregistered prior to the research being undertaken.

\section{RESULTS}

\subsection{Demographic and clinical variables}

Demographic, clinical, and neuropsychological scores for all participants are presented in Table 1. The LPA, AD, and Control groups did not differ in terms of sex distribution $(p=.47)$, age $(p=.38)$, and education $(p=.24)$. Importantly, the LPA and AD groups were matched for age at disease onset $(p=.77)$, disease severity (CDR-FTLD SoB: $p$ $=.34$ ), and clinician-indexed memory impairment (CDR-FTLD Memory subdomain) $(p=.18)$. LPA and AD groups were further comparable on carer-reported changes in behaviour and memory (CBI-R total: $p=.62$, and CBI-R memory component: $p=.50$ ). Significant group effects were noted on the ACE-R total score, with both patient groups performing significantly lower relative to Controls (both $p$ values $<.001$ ). Importantly, no significant differences were evident between patient groups for global cognitive function on the ACE-R $(p=.42)$.

\subsection{Neuropsychological test performance}


Amnesia in Logopenic Progressive Aphasia

Formal neuropsychological testing revealed characteristic cognitive profiles in the LPA and AD groups (Table 1). Relative to Controls, LPA patients displayed marked impairments on global measures of language function, as well as on targeted tests of naming, comprehension, repetition, executive function, sustained attention, working memory, verbal fluency, and visuo-constructional abilities (all $p$ values $<.001$ ). Similarly, the AD group displayed canonical impairments on global tests of memory and language, as well as specific tests of naming, comprehension, executive function, sustained attention, working memory, verbal fluency, and visuo-constructional abilities relative to the Control group (all $p$ values $<.001$ ). Direct comparisons of the patient groups revealed disproportionate impairment of single word repetition in the LPA relative to the AD group ( $p=.006)$, consistent with the clinical phenotype of LPA. On all other neuropsychological test measures, the LPA and AD groups performed comparably (all $p$ values $\geq .07$ ). These cognitive profiles are in keeping with earlier descriptions of LPA (Butts et al., 2015; Magnin et al., 2013) and AD (Graham, Emery, \& Hodges, 2004; Hutchinson \& Mathias, 2007; Ramanan et al., 2017).

[INSERT TABLE 1 HERE]

\subsection{Episodic memory assessment}

\subsubsection{Delayed recall performance}

Group differences on episodic memory tests are displayed in Fig. 1 and Table 2. Significant group effects were observed for verbal (RAVLT 30-mins delayed recall: $p$ $<.001$ ) and nonverbal (ROCF \% retained: $p<.001$ ) delayed recall. Irrespective of modality, LPA and AD groups performed significantly poorer relative to Controls (all $p$ values $<.01)$. While LPA patients outperformed the AD group on the verbal delayed 
Amnesia in Logopenic Progressive Aphasia

recall measure $(p=.03)$, no significant difference was observed between patient groups for nonverbal delayed recall $(p=.51)$. Together, these findings corroborate previous reports of disrupted memory performance in LPA relative to healthy Controls (Ramanan et al., 2016; Win et al., 2017).

[INSERT FIGURE 1 HERE]

[INSERT TABLE 2 HERE]

\subsubsection{Correlations with neuropsychological task performance}

No significant correlations emerged between verbal or nonverbal episodic recall and neuropsychological test performance, including targeted neuropsychological assessments of language, in LPA (all $p$ values $>.1$ ) or $\mathrm{AD}$ (all $p$ values $\geq .08$ ) (Supplementary Table 2).

\subsection{VBM analyses}

\subsubsection{Group differences in grey matter intensity}

Group differences in grey matter intensity are presented in Supplementary Table 3 and Supplementary Fig. 1. Relative to Controls, the LPA group displayed reduced grey matter intensity predominantly in left posterior temporoparietal regions including the left AG and supramarginal gyri, bilateral inferior/middle/superior temporal gyri (left > right), bilateral temporal poles (left $>$ right), and the left hippocampus (across the longitudinal axis). These patterns are in keeping with previous descriptions of LPA (Gorno-Tempini et al., 2004; Rogalski et al., 2014). By contrast, the AD group displayed widespread bilateral atrophy including the hippocampi, medial and lateral 
Amnesia in Logopenic Progressive Aphasia

temporal and parietal, and prefrontal regions, in line with previous descriptions (Karas et al., 2004; Moller et al., 2013).

Direct comparisons between patient groups failed to reveal any significant clusters at $p$ $<.005$ corrected for Family-Wise Error.

\subsubsection{Grey matter correlates of delayed recall performance}

Associations between episodic delayed recall performance and regions of significant grey matter intensity decrease in patient groups relative to Controls are displayed in Fig. 2 and 3, Table 3, and Supplementary Tables 4 and 5.

[INSERT FIGURE 2 HERE]

\subsubsection{LPA group}

Verbal episodic memory performance in LPA correlated with grey matter intensity in the left hippocampus, bilateral posterior parietal (including AG), lateral temporal, medial and lateral prefrontal regions (Supplementary Table 4; Fig. 2). By contrast, nonverbal episodic memory correlated with reduced grey matter intensity in the bilateral $\mathrm{AG}$ and frontal poles, left orbitofrontal cortex, and left precuneus (Supplementary Table 5, Fig. 3).

Irrespective of modality, episodic memory performance in the LPA group was associated with grey matter intensity reduction in the bilateral AG, left orbitofrontal cortex and left postcentral gyrus (Supplementary Fig. 2 and Supplementary Table 6). 
Amnesia in Logopenic Progressive Aphasia

\subsubsection{AD group}

Verbal delayed recall performance in $\mathrm{AD}$ was associated with grey matter intensity in bilateral medial and lateral temporal structures, bilateral posterior parietal (including AG), medial and lateral prefrontal regions, as well as bilateral insular cortices and paracingulate gyri (Supplementary Table 4; Fig. 2). By contrast, nonverbal episodic delayed recall was associated with reduced grey matter intensity in the bilateral AG and frontal poles, right posterior cingulate cortex, and left middle temporal gyrus (Supplementary Table 5, Fig. 3).

Episodic memory impairments (regardless of modality) in AD correlated with reduced grey matter intensity in the bilateral AG, left inferior/middle/superior temporal gyri, left insular cortex, and right frontal pole (Supplementary Fig. 2 and Supplementary Table 6).

[INSERT TABLE 3 HERE]

[INSERT FIGURE 4 HERE]

\subsubsection{Disease-general neural correlates}

Across LPA and AD groups, verbal delayed recall impairments were associated with reduced grey matter intensity in the left hippocampus, bilateral AG, bilateral frontal poles, bilateral inferior/middle/superior temporal gyri, and the left middle frontal gyrus (Table 3, Fig. 4). Nonverbal episodic memory impairments were commonly associated 
Amnesia in Logopenic Progressive Aphasia

with reduced grey matter intensity in the bilateral AG, right frontal pole, and left orbitofrontal cortex in both patient groups (Table 3, Fig. 4).

\subsection{Diffusion tensor imaging analyses}

\subsubsection{Group differences in FA}

Characteristic patterns of FA decrease relative to Controls were observed in LPA and AD (Supplementary Table 7). LPA patients displayed predominantly left-lateralized FA reduction in the inferior and superior longitudinal fasciculi, inferior fronto-occipital fasciculus, and forceps major bundles. By contrast, the AD group displayed widespread bilateral FA reductions in the cingulum bundle, superior and inferior longitudinal fasciculi, inferior fronto-occipital fasciculi, and forceps major bundles. Together, these patterns are in keeping with earlier descriptions of FA reductions in LPA (Tu et al., 2016) and AD (Agosta et al., 2011; Villain et al., 2008).

Direct comparisons between patient groups failed to reveal any significant clusters at $p$ $<.005$ corrected for Family-Wise Error.

[INSERT FIGURE 5 HERE]

\subsection{Probabilistic fibre tracking analyses}

\subsubsection{Group differences in tract-of-interest microstructure}

Fig. 5A depicts an exemplar 2D/3D reconstructed left AG-hippocampal complex inferior longitudinal fasciculus (ILF) tract from the probabilistic tractography analyses of a single healthy Control subject. Group differences for tract metrics for the tract-ofinterest are displayed in Supplementary Table 8. Both patient groups displayed significantly higher MD relative to the Control group (all $p$ values $<.01$ ), indicating 
Amnesia in Logopenic Progressive Aphasia

reduced microstructural integrity in the tract-of-interest. Importantly, no significant differences were noted between LPA and AD groups on this index $(p>.1)$. No significant group differences emerged on the FA metric for the tract-of-interest $(p>.1)$.

For the tract-of-no-interest (i.e., left corticospinal tract), the AD group demonstrated increased MD $(p=.02)$, relative to the Control group. No other significant group differences emerged on tract metrics for the tract-of-no-interest (all $p$ values $>.1$ ).

\subsubsection{Correlations between tract-of-interest metrics and episodic memory}

Fig. 5B-5C displays significant correlations that emerged between tract metrics from the modelled left AG-hippocampal ILF tract and episodic delayed recall measures for the LPA and AD contrasts.

For the LPA contrast, significant correlations were found between verbal delayed recall performance and the MD metric (Fig. 5B: $r=-.38 ; p=.01)$, but not FA $(r=.01 ; p>.1)$. By contrast, no significant correlations emerged between either FA or MD metrics and nonverbal episodic recall performance (both $r$ values $\leq .-.29$; both $p$ values $>.07$ ).

Similarly, for the AD contrast, significant correlations emerged between verbal delayed recall performance and the MD metric (Fig. $5 \mathrm{C}: r=-.52 ; p<.001)$ but not the FA metric $(r=-.09 ; p>.1)$. No significant correlations emerged between either FA or MD metrics and nonverbal episodic recall performance (both $r$ values $\leq-.17$; both $p$ values $>.1$ ).

\subsubsection{Correlations between tract-of-no-interest metrics and episodic memory}


Amnesia in Logopenic Progressive Aphasia

Finally, in line with our predictions, no significant correlations emerged between either episodic delayed recall measure and any of the tract metric values in the tract-of-nointerest (i.e., left corticospinal tract) for either contrasts (LPA: all $r$ values $\leq-.24$; all $p$ values $>.1$; AD: all $r$ values $\leq-.22$; all $p$ values $>.1$ ).

\section{DISCUSSION}

Mounting evidence suggests the presence of marked verbal and nonverbal episodic memory impairments in LPA. To our knowledge, this is the first study to explore the grey and white matter underlying neural correlates of these memory impairments in LPA in contrast with typical AD. Verbal memory deficits in LPA were observed at an intermediate level between typical AD and healthy Controls, reflecting a gradation of memory impairment across the AD-LPA spectrum. Despite previous suggestions that verbal episodic memory impairments in LPA manifest due to prominent language and lexical retrieval disturbances characteristic of this syndrome (Win et al., 2017), our correlation analyses failed to reveal significant associations between verbal episodic memory performance and a confrontational naming/lexical retrieval task. Moreover, our observation of prominent nonverbal episodic memory dysfunction in LPA suggests that language impairment is not the primary mechanism underpinning memory disturbance in this syndrome. In fact, on a test of delayed nonverbal memory that circumvents language demands, LPA patients were indistinguishable from matched cases of typical AD. Finally, objective memory impairments in LPA were corroborated by clinician-indexed and carer-reported difficulties, again of a comparable magnitude as that reported in the $\mathrm{AD}$ group. Collectively, these findings indicate a pervasive memory impairment in LPA evident not only on objective neuropsychological tests but manifest in the everyday activities of LPA patients. 
To further understand the origins of memory dysfunction in LPA, we conducted multimodal neuroimaging analyses exploring grey and white matter contributions to task performance. Considering first the grey matter patterns of atrophy, our VBM analyses revealed that in addition to left perisylvian atrophy, our LPA cohort demonstrated significant left hippocampal atrophy. The hippocampus, however, was implicated only for verbal episodic memory performance in our covariate analyses. By contrast, the left inferior parietal cortex was found to correlate with episodic memory dysfunction in LPA, irrespective of modality, suggesting an important modulating role of parietal regions in the genesis of memory dysfunction in this syndrome (Casaletto et al., 2017; Krishnan et al., 2017). Notably, we also found evidence of left orbitofrontal cortex involvement in episodic memory dysfunction in LPA. This finding was surprising as orbitofrontal and prefrontal regions are typically affected later in the LPA disease course (Rohrer et al., 2013). The emergence of the orbitofrontal regions in our VBM analyses may reflect differences in the disease severity of our LPA cohort relative to previous reports (Teichmann et al., 2013; Win et al., 2017). Nevertheless, our findings reveal pervasive episodic memory impairments in LPA (Eikelboom et al., 2018), attributable to erosion of large-scale predominantly left-sided brain networks implicated in language and memory processing.

Turning our attention to the $\mathrm{AD}$ group, our covariate analyses pointed to grey matter intensity decrease in bilateral medial temporal, inferior parietal, and prefrontal regions as driving episodic memory dysfunction, with the notable involvement of the posterior cingulate cortex. Episodic memory dysfunction has long been heralded as the cognitive signature of typical AD, traditionally thought to reflect early MTL dysfunction 
(Dickerson \& Eichenbaum, 2010). Recent evidence, however, suggests that in addition to MTL damage, network-wide degeneration of the posterior cingulate, inferior parietal, lateral temporal, and prefrontal cortices gives rise to the hallmark amnesic profile in AD (Desgranges et al., 2002; Irish et al., 2016; Irish et al., 2014; Ramanan et al., 2019). The emergence of inferior parietal and prefrontal regions as common substrates of memory dysfunction in LPA and $\mathrm{AD}$, further reinforces the importance of regions beyond the MTL in supporting episodic memory processes and highlights a specific vulnerability of parietal cortical nodes of the episodic memory network across the AD spectrum.

To complement the grey matter VBM analyses, indices of white matter microstructural integrity were employed to further understand how disruption of structural connectivity between nodes of the core memory network contributes to episodic amnesia in LPA and $\mathrm{AD}$. In both patient groups, large-scale degeneration of subcortical pathways that connect parieto-occipital, frontal, and lateral temporal/MTL regions was evident. In keeping with the LPA clinical phenotype, these disruptions were largely left-lateralized reflecting subcortical disconnections from parietal to frontotemporal regions, along the perisylvian language pathway (Galantucci et al., 2011; Tu et al., 2016). Long-range fibres such as the ILF have been shown to relay information between parieto-occipital and temporal lobes facilitating successful lexical retrieval and episodic memory processing (Herbet, Zemmoura, \& Duffau, 2018). Unlike LPA, however, our typical AD cohort displayed diffuse white matter damage to bilateral subcortical fibre tracts. Unique to $\mathrm{AD}$ was the disruption of the cingulum bundle, which runs medially to the ILF to connect the MTL with an important posterior cortical memory hub - the posterior cingulate cortex (Catani et al., 2002). The posterior cingulate cortex holds a 
Amnesia in Logopenic Progressive Aphasia

topologically central role in anchoring multiple structural brain networks (Hagmann et al., 2008) and its degeneration in $\mathrm{AD}$ has been uniquely linked to deficits in episodic recollection and related constructive endeavours (Irish, Addis, Hodges, \& Piguet, 2012; Irish et al., 2015; Ramanan et al., 2019).

Considering next how structural white matter alterations impact memory performance in LPA, our probabilistic tractography analyses isolated a subsection of the ILF that has been well described in the past to form structural connections between the left AG and left MTL regions (Caspers et al., 2011; Rushworth et al., 2006; Uddin et al., 2010). Interestingly, associations between tract integrity and memory performance were restricted to verbal episodic recall performance in both patient groups. This finding resonates with the proposal that the left ILF supports communication between areas specialized for processing verbal content (Kelley et al., 1998). Our results therefore suggest an important role for subcortical white matter tract degeneration in the origin of episodic memory dysfunction in LPA and AD. Structural and functional connections between the MTL and inferior parietal cortex play an important mediating role in the service of successful episodic recollection (Gilmore, Nelson, \& McDermott, 2015; Ramanan et al., 2019; Vincent et al., 2006). While the left ILF was implicated in both LPA and AD, we tentatively speculate as to the temporal origins of microstructural damage to this tract. Our cross-sectional design precludes the direct examination of evolution of disease pathology along white matter tracts, however, we suggest that typical AD pathology propagates from the MTL back to the parietal cortex (Khan et al., 2014), whereas the LPA syndrome may unfold in the converse direction with MTL hypometabolism emerging as secondary to the downstream propagation of pathology from the temporoparietal cortex. Whether the ILF represents a preferential subcortical 
Amnesia in Logopenic Progressive Aphasia

pathway for the encroachment of pathology in LPA is an important question to address via longitudinal studies incorporating multimodal grey and white matter neuroimaging metrics.

The findings reported here should be interpreted in relation to a number of methodological considerations. First, our relatively modest sample size of LPA patients reflects the rarity of this syndrome, and constrained our tractography analyses, whereby we were unable to examine within group correlations due to concerns of reduced statistical power. Next, the verbal and nonverbal episodic memory measures used in the current study differed considerably on administration and scoring procedures, number of learning trials provided and the length of filled delay, possibly amounting to both measures tapping into different aspects of episodic memory. Importantly, these measures were chosen as they are included as part of routine neuropsychological assessment in our clinic. Despite both tests being widely adopted and standardized indices of episodic memory, future studies will benefit from the inclusion of comprehensive episodic memory measures with verbal and nonverbal components that are comparable on administration, methodological, and scoring procedures. A good example of this is the Hopkins Verbal Learning Test - Revised and its visual counterpart, the Brief Visual Memory Test - Revised. Both measures are methodologically similar and involve a series of verbal or visual items presented over three repeated trials, a delayed recall test following a 25-minute delay, and a recognition test (Strauss et al., 2006). Future studies should consider the usage of methodologically comparable measures providing a comprehensive impression of cross-modal memory performance in patients with neurodegenerative syndromes. Further, a majority of our LPA patients have not yet come to autopsy and have no pathological confirmation. 
Related to this, our LPA patients may represent a more clinically advanced cohort in relation to previous studies. It will be important to replicate and extend these findings in a larger group of LPA patients at different stages of disease severity. Moreover, longitudinal investigations charting the evolution of memory impairments over time, and their respective neural bases, will be invaluable. In light of recent suggestions for heterogenous cognitive and atrophy profiles within homogenously classified LPA cohorts (Leyton et al., 2015), future studies may benefit from examining whether episodic memory deficits in LPA represent a unifying factor across distinct endophenotypes of this syndrome.

Despite these caveats, our findings hold a number of important clinical implications, which warrant attention. The presence of verbal and nonverbal episodic memory dysfunction in LPA runs counter to its conceptualisation as primarily a disorder of language and may unwittingly predispose a clinician to confer a diagnosis of typical $\mathrm{AD}$ with language features. This risk is further increased when such memory impairments arise in the context of positive amyloid profiles on PET-ligand neuroimaging. The differential diagnosis of LPA from other primary progressive aphasias hinges on distinguishing between distinct language profiles (Gorno-Tempini et al., 2011), yet classification based on language performance produces false positive rates, as high as $14 \%$, in distinguishing LPA from other primary progressive aphasias (Savage et al., 2013). By contrast, the early presence of nonverbal episodic amnesia, in the context of other primary progressive aphasias, appears to be unique to LPA (Ramanan et al., 2016), suggesting that conjunctive reliance on language and episodic memory performance may significantly improve the accurate diagnosis of LPA. Future work tracking the emergence of memory disturbances at the earliest stages of the 
Amnesia in Logopenic Progressive Aphasia

syndrome employing longitudinal approaches in conjunction with PET-ligand neuroimaging will prove particularly informative in this regard.

In conclusion, the current findings demonstrate the presence of stark verbal and nonverbal episodic memory deficits in LPA reflecting the degeneration of medial temporal, inferior parietal and prefrontal cortical regions, as well as disrupted white matter connectivity between inferior parietal and MTL regions. Our findings suggest that the current heuristic of diagnosing LPA predominantly based on language impairments fails to account for the inherent variability in cognitive profiles displayed across the disease trajectory. We suggest that adopting a multidimensional approach to understanding cognitive trajectories in LPA will greatly improve diagnosis and management of this syndrome.

\section{Acknowledgements}

The authors are grateful to the patients and families for their continued support of our research. The authors wish to acknowledge the Sydney Informatics Hub funded by the University of Sydney for providing access to High Performance Computing (HPC) facilities.

\section{Funding}

This work was supported in part by funding to Forefront, a collaborative research group specialised to the study of frontotemporal dementia and motor neurone disease, from the National Health and Medical Research Council (NHMRC) of Australia program grant (APP1037746) and the Australian Research Council (ARC) Centre of Excellence in Cognition and its Disorders Memory Program (CE110001021). Siddharth Ramanan 
Amnesia in Logopenic Progressive Aphasia

is supported by a Faculty of Science Ph.D. Research Scholarship from The University of Sydney. Lars Marstaller is supported by the European Union Horizon 2020 research and innovation programme under the Marie Skłodowska-Curie grant (agreement no. 663830). Olivier Piguet is supported by an NHMRC Senior Research Fellowship (APP1103258). Muireann Irish is supported by an ARC Future Fellowship (FT160100096) and an ARC Discovery Project (DP180101548).

\section{Competing interests}

The authors report no competing interests.

\section{Supplementary material}

One supplementary file with supplementary methods, eight supplementary tables, and two supplementary figures. 
Amnesia in Logopenic Progressive Aphasia

Table 1. Demographic, clinical, and general neuropsychological assessment performance for all groups.

\begin{tabular}{|c|c|c|c|c|c|}
\hline & LPA & $\mathrm{AD}$ & Controls & $\begin{array}{l}\text { Magnitude of } \\
\text { group effect }^{\ddagger}\end{array}$ & $\begin{array}{ll}\text { LPA } & \text { vs. } \\
\text { AD } \quad(p\end{array}$ \\
\hline & & & & & $\begin{array}{l}\text { value and } \\
\text { effect size) }\end{array}$ \\
\hline$N$ & 19 & 23 & 31 & & \\
\hline $\operatorname{Sex}(M: F)$ & $8: 11$ & $12: 11$ & $11: 20$ & $\chi^{2}=4.4 ; p=.47$ & \\
\hline Age (years) & $\begin{array}{l}69.6 \\
(7.40)\end{array}$ & $\begin{array}{l}69.5 \\
(8.0)\end{array}$ & $\begin{array}{l}71.6 \\
(2.2)\end{array}$ & $\begin{array}{l}F=.9 ; p=.38 ; \eta_{p}^{2} \\
=.02\end{array}$ & $.82 ; d=.01$ \\
\hline Education (years) & $13.5(3.6)$ & $\begin{array}{l}12.5 \\
(2.8)\end{array}$ & $\begin{array}{l}12.2 \\
(1.8)\end{array}$ & $\begin{array}{l}F=1.4 ; p \\
=.24 ; \eta_{p}^{2}=.03\end{array}$ & $.60 ; d=.31$ \\
\hline $\begin{array}{l}\text { Age of disease onset } \\
\text { (years) }\end{array}$ & $62.3(7.7)$ & $\begin{array}{l}61.6 \\
(8.1)\end{array}$ & - & $t=-.28 ; p=.77$ & $.77 ; d=.09$ \\
\hline $\begin{array}{l}\text { Disease severity } \\
\text { (CDR-FTLD SoB) }\end{array}$ & $5.1(3.3)$ & $3.9(2.5)$ & - & $W=130.5 ; p=.34$ & $.34 ; d=.76$ \\
\hline $\begin{array}{l}\text { Clinician-rated } \\
\text { memory }\end{array}$ & $.5(.5)$ & $.6(.3)$ & - & $W=201 ; p=.18$ & $\begin{array}{l}.18 ; d= \\
-.25\end{array}$ \\
\hline impairment (CDR- & & & & & \\
\hline $\begin{array}{l}\text { FTLD Memory } \\
\text { subscore) }\end{array}$ & & & & & \\
\hline CBI-R Total (\%) & $\begin{array}{l}19.9 \\
(11.8)\end{array}$ & $\begin{array}{l}16.1 \\
(9.9)\end{array}$ & $3.0(3.0)$ & $\begin{array}{l}F=25 ; p<.001 \\
\eta_{p}^{2}=.43\end{array}$ & $.62 ; d=.35$ \\
\hline
\end{tabular}


Amnesia in Logopenic Progressive Aphasia

$\begin{array}{lllllllll}\text { CBI-R Memory (\%) } & 42.6 & 37.3 & 6.2(6.8) & F=45.1 ; \quad p & .50 ; d=.3 \\ & (17.4) & (17.5) & & <.001 ; \eta_{p}^{2}=.58 & \\ \text { ACE-R total (100) } & 71.6 & 76.6 & 95.3 & F=72.8 ; \quad \boldsymbol{p} .42 ; \quad d= \\ & (11.2) & (7.5) & (3.3) & <. \mathbf{0 0 1} ; \eta_{p}^{2}=.67 & -.55\end{array}$

Neuropsychological

tests

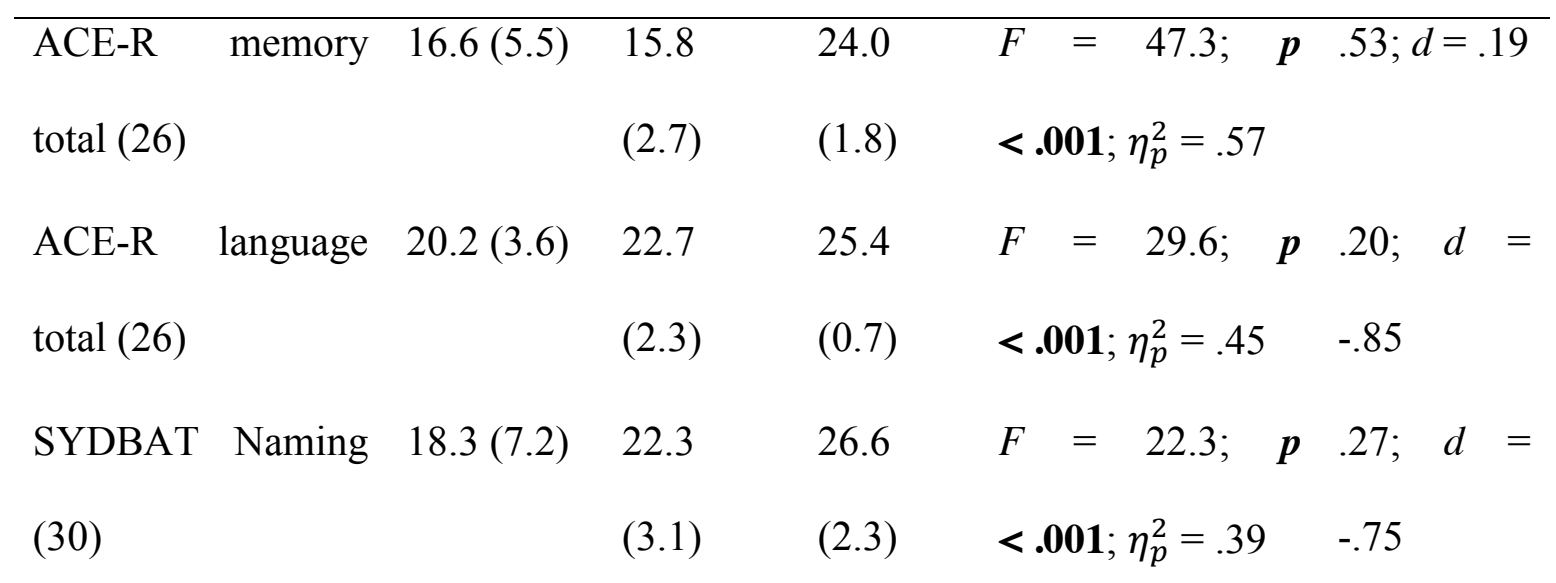

$\begin{array}{llllll}\text { SYDBAT } & 26.3(1.8) & 26.4 & 29.1 & F= & 15.3 ; \boldsymbol{p} .64 ; \quad d=\end{array}$

Comprehension $\quad(2.7) \quad(1.3) \quad<.001 ; \eta_{p}^{2}=.31 \quad-.04$

$(30)$

SYDBAT

Repetition (30)

$28.9(0.9) \quad 29.5$

$(0.9)$

167.8

(189.1) (70.6)

Digit span forward $7.6(1.9)$

(16)

Digit

span $4.7(1.7)$

backward (16)
$9.3(1.8) \quad 11.1 \quad F=17.4 ; \quad p \quad .07 ; \quad d=$

(2.1) $\quad<.001 ; \eta_{p}^{2}=.33 \quad-.92$

$5.2(1.9) 7.4(2.2) \quad F=12.6 ; \quad p \quad .49 ; \quad d=$ $<.001 ; \eta_{p}^{2}=.26 \quad-.28$ 
Amnesia in Logopenic Progressive Aphasia

$\begin{array}{llllll}\text { Letter fluency (FAS } & 25.7 & 29.6 & 45.5 & F=19.8 ; \quad \boldsymbol{p} .46 ; \quad d= \\ \text { total) } & (11.6) & (12.2) & (11.7) & <.001 ; \eta_{p}^{2}=.36 & -.33 \\ \text { ROCF copy (36) } & 26.6(7.9) & 24.4 & 32.5 & F=8.7 ; \boldsymbol{p}<.001 ; .78 ; d=.24 \\ & & (10.4) & (2.9) & \eta_{p}^{2}=.20\end{array}$

Note. For all tests/variables, maximum scores reported in brackets; For all groups, mean and standard deviation reported; For magnitude of group effect, $p$-values and $\quad{ }_{p}^{2}$ values, along with the accompanying $t / W / F$ values reported; ${ }^{*}$ For all $F$-statistics, $\mathrm{df}_{\text {numerator }}=2$ and $\mathrm{df}_{\text {denominator }}=70$; For all statistical outputs, exact $p$ and $\eta_{p}^{2}$ values reported; For all post-hoc comparisons, exact $p$ values reported; all $p$ values bolded if below $p<.05$; effect size for between patient post-hoc comparison calculated using Cohen's $d$; LPA $=$ Logopenic Progressive Aphasia; $\mathrm{AD}=$ Alzheimer's disease; CDR-FTLD SoB = Clinical Dementia Rating - Frontotemporal Lobar Degeneration Sum of Boxes; CBI$\mathrm{R}=$ Cambridge Behavioural Inventory - Revised; ACE- $\mathrm{R}=$ Addenbrooke's Cognitive Examination - Revised; SYDBAT = Sydney Language Battery; TMT B-A = time difference between parts B and A of the Trail Making Test; ROCF = Rey-Osterrieth Complex Figure. 
Amnesia in Logopenic Progressive Aphasia

Table 2. Delayed episodic memory performance across participant groups

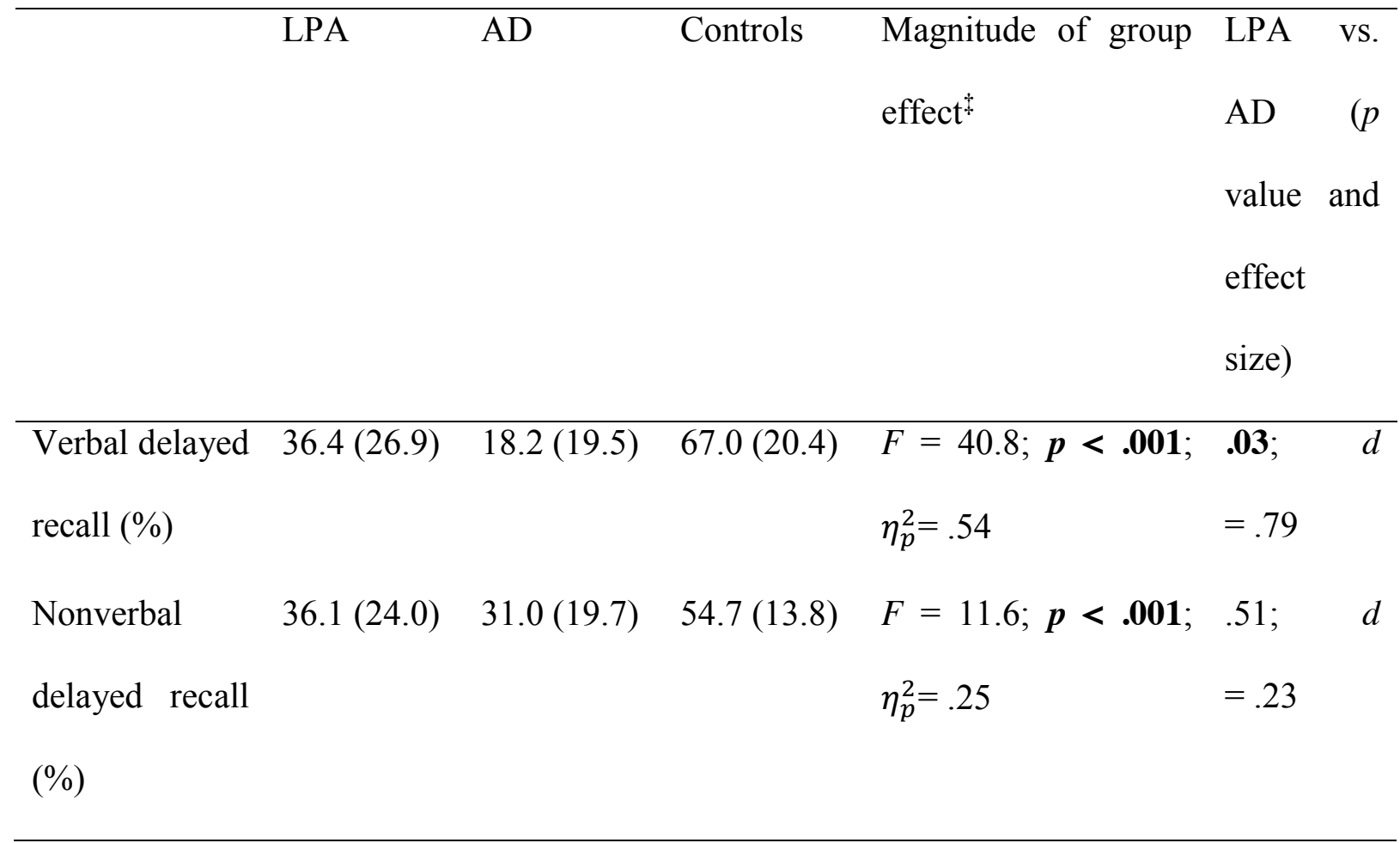

Note. For magnitude of group effect, $F, p$, and $\eta_{p}^{2}$ values are reported; ${ }^{\dagger}$ For all $F$ statistics, $\mathrm{df}_{\text {numerator }}=2$ and $\mathrm{df}_{\text {denominator }}=69$; For all post-hoc comparisons, exact $p$ values reported; all $p$ values bolded if below $p<.05$; effect size for between patient post-hoc comparison calculated using Cohen's $d$; Verbal delayed recall was indexed using the Rey Auditory Verbal Learning Test 30-minute recall percent score while nonverbal delayed recall was measured using the Rey Osterrieth Complex Figure percentage retained score $\mathrm{LPA}=$ Logopenic Progressive Aphasia; $\mathrm{AD}=$ Alzheimer's disease. 
Amnesia in Logopenic Progressive Aphasia

Table 3. Voxel-based morphometry results indicating grey matter regions commonly implicated in LPA and AD groups for episodic delayed recall performance

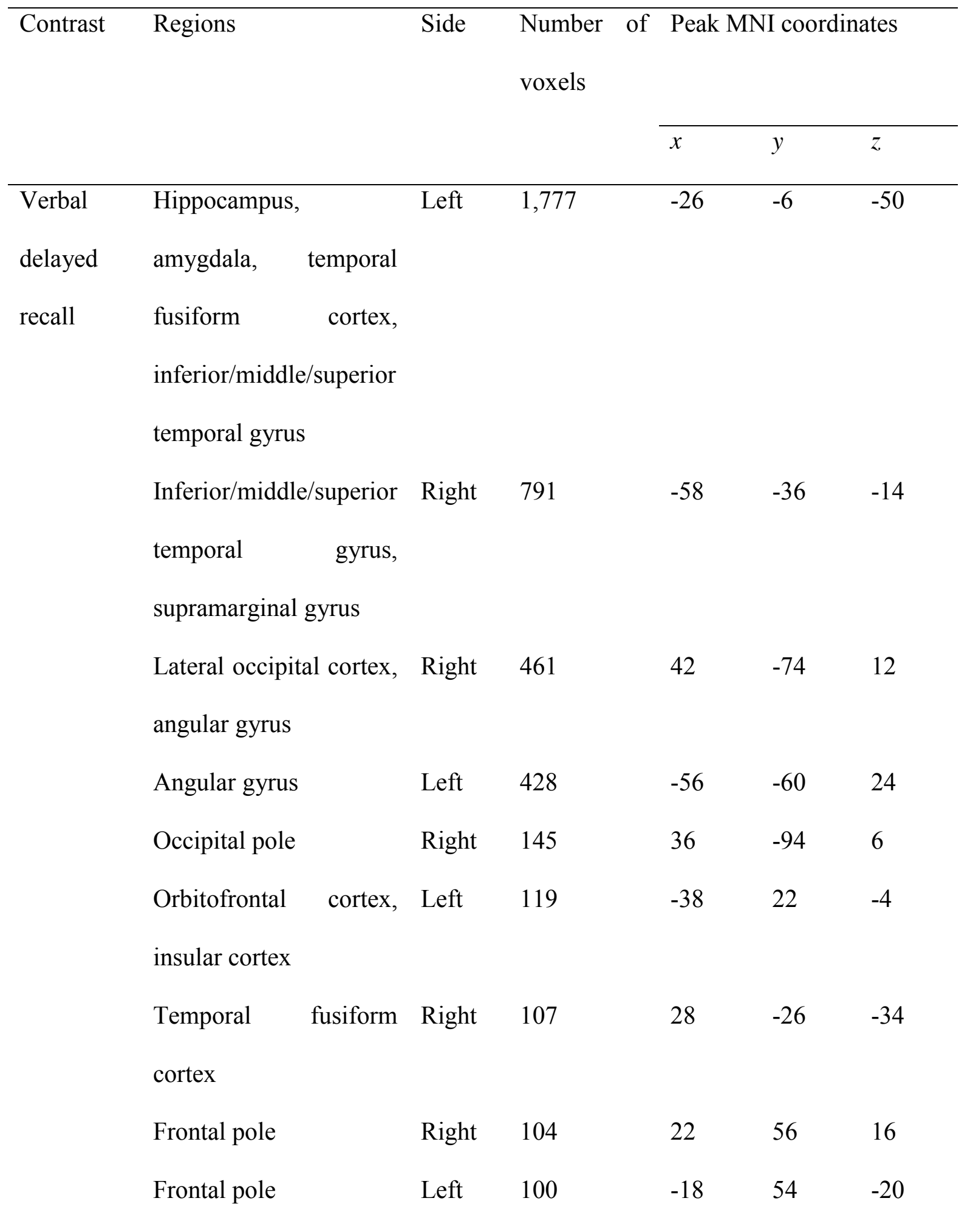




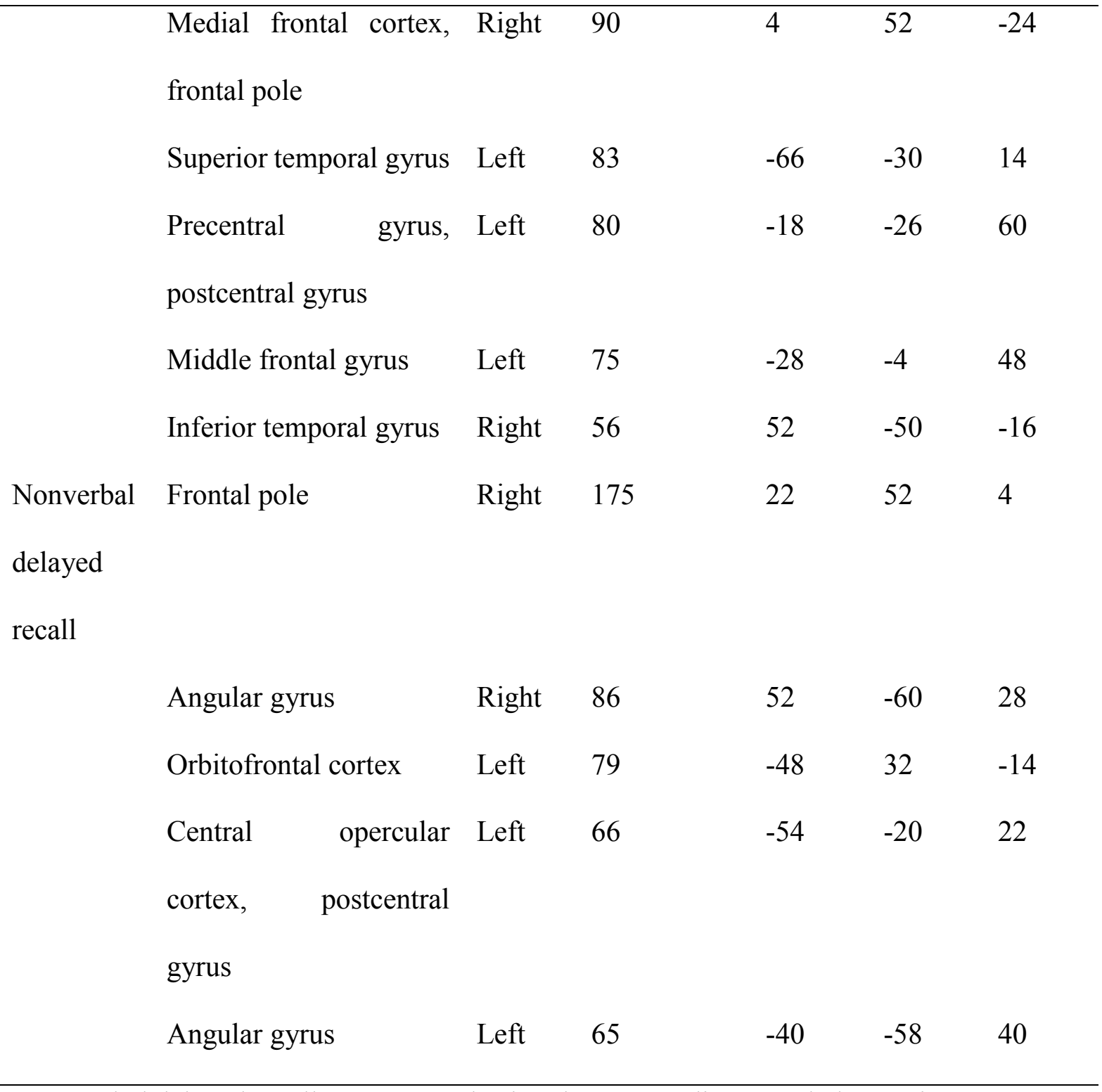

Note. Verbal delayed recall was assessed using the Rey Auditory Verbal Learning Test 30-minute recall percent score and nonverbal delayed recall was indexed using the Rey Osterrieth Complex Figure percentage retained score. All clusters reported using voxelwise contrasts corrected using False Discovery Rate at $p<.05$. Age is included as a covariate in all contrasts. MNI, Montreal Neurological Institute; LPA = Logopenic Progressive Aphasia; AD = Alzheimer's disease. 
Amnesia in Logopenic Progressive Aphasia

Fig. 1. Episodic memory performance for all groups on the verbal and nonverbal delayed recall measures. Boxes depict distribution of data with lower and upper end of the box depicting the inter-quartile range respectively. The bolded horizontal lines depict the median score while whiskers depict the variability outside the upper and lower quartiles. Verbal delayed recall assessed via the Rey Auditory Verbal Learning Test 30-minute recall percent score and nonverbal delayed recall assessed using the Rey Osterrieth Complex Figure percentage retained score; LPA = Logopenic Progressive Aphasia; $\mathrm{AD}=$ Alzheimer's disease. 
Amnesia in Logopenic Progressive Aphasia

Fig. 2. Regions of significant grey matter intensity decrease that correlate with verbal delayed recall performance in LPA and AD. Correlation analyses in both contrasts were run in a combined patient-Control group (i.e., LPA and Controls, and AD and Controls). Coloured voxels indicate regions that emerged significant in the voxel-based morphometry analyses at $p<.05$ corrected for False Discovery Rate with a cluster threshold of 100 contiguous voxels. All clusters reported at $t \geq 3.1$ (see Supplementary Table 4 for magnitude of $t$-values for each cluster). Age was included as a covariate in the analyses. Clusters are overlaid on the Montreal Neurological Institute (MNI) standard brain with $x$ and $y$ coordinates reported in MNI standard space. L = Left; LPA = Logopenic Progressive Aphasia; AD = Alzheimer's disease. 
Amnesia in Logopenic Progressive Aphasia

Fig. 3. Regions of significant grey matter intensity decrease that correlate with nonverbal delayed recall performance in LPA and AD. Correlation analyses in both contrasts were run in a combined patient-Control group (i.e., LPA and Controls, and $\mathrm{AD}$ and Controls). Coloured voxels indicate regions that emerged significant in the voxel-based morphometry analyses at $p<.05$ corrected for False Discovery Rate with a cluster threshold of 100 contiguous voxels. All clusters reported at $t \geq 3.37$ (see Supplementary Table 5 for magnitude of $t$-values for each cluster). Age was included as a covariate in the analyses. Clusters are overlaid on the Montreal Neurological Institute (MNI) standard brain with $x$ and $y$ coordinates reported in MNI standard space. $\mathrm{L}=$ Left; LPA = Logopenic Progressive Aphasia; AD = Alzheimer's disease. 
Amnesia in Logopenic Progressive Aphasia

Fig. 4. Inclusive masking results indicating disease-general brain regions associated with verbal and nonverbal episodic delayed recall performance. Inclusive masks were derived from correlation analyses run in a combined patient-Control group (i.e., LPA and Controls, and $\mathrm{AD}$ and Controls), separately for verbal and nonverbal episodic delayed recall performance. Coloured voxels indicate regions that emerged significant in the voxel-based morphometry analyses at $p<.05$ corrected for False Discovery Rate. All clusters reported at $t \geq 3.10$ (see Supplementary Table 4 and 5 for magnitude of $t$ values for each cluster). Age was included as a covariate in the analyses. Clusters are overlaid on the Montreal Neurological Institute (MNI) standard brain with $x$ and $y$ coordinates reported in MNI standard space. $\mathrm{L}=$ Left; LPA = Logopenic Progressive Aphasia; $\mathrm{AD}=$ Alzheimer's disease. 
Amnesia in Logopenic Progressive Aphasia

Fig. 5. Correlations between extracted ILF tractmetrics and verbal episodic delayed recall measures for both patient groups. Upper panel shows an exemplar 2D/3D reconstruction of the modelled left angular gyrus-hippocampal inferior longitudinal fasciculus (ILF) tract for a single Control subject, with indications of the neuroanatomical position of the angular gyrus and hippocampus relative to the modelled tract. Lower panel indicates significant correlations (Pearson's $r$ coefficients and $p$-values) that emerged between MD values for the extracted ILF and episodic delayed recall measures for LPA and Control participants combined, where higher tractmetric values indicate greater white matter microstructural damage; MD values are measured in $\mathrm{mm}^{2} / \mathrm{sec} \times 10^{-3}$; Verbal delayed recall was assessed using the Rey Auditory Verbal Learning Test 30-minute delayed recall score (expressed as percentage) and nonverbal delayed recall score was assessed using the Rey Complex Figure Test 3minute percentage retained score; $\mathrm{L}=$ Left; $\mathrm{R}=$ Right; $\mathrm{MD}$, mean diffusivity; $\mathrm{LPA}=$ Logopenic Progressive Aphasia; AD = Alzheimer's disease. 


\section{References}

Agosta, F., Pievani, M., Sala, S., Geroldi, C., Galluzzi, S., Frisoni, G. B., \& Filippi, M. (2011). White Matter Damage in Alzheimer Disease and Its Relationship to Gray Matter Atrophy. Radiology, 258(3), 853-863. doi: 10.1148/radiol.10101284

Ahmed, S., de Jager, C. A., Haigh, A. M., \& Garrard, P. (2012). Logopenic aphasia in Alzheimer's disease: clinical variant or clinical feature? J Neurol Neurosurg Psychiatry, 83(11), 1056-1062. doi: 10.1136/jnnp-2012-302798

Andersson, J. L. R., Jenkinson, M., \& Smith, S. (2007a). Non-linear optimisation FMRIB Technical Report TR07JA1. Oxford: University of Oxford FMRIB Centre.

Andersson, J. L. R., Jenkinson, M., \& Smith, S. (2007b). Non-linear registration, aka spatial normalisation FMRIB Technical Report TRO7JA2. Oxford: University of Oxford FMRIB Centre.

Benjamini, Y., \& Hochberg, Y. (1995). Controlling the False Discovery Rate - a Practical and Powerful Approach to Multiple Testing. Journal of the Royal Statistical Society Series B-Methodological, 57(1), 289-300.

Berryhill, M. E. (2012). Insights from neuropsychology: pinpointing the role of the posterior parietal cortex in episodic and working memory. Front Integr Neurosci, 6, 31. doi: 10.3389/fnint.2012.00031

Bertoux, M., de Souza, L. C., Corlier, F., Lamari, F., Bottlaender, M., Dubois, B., \& Sarazin, M. (2014). Two distinct amnesic profiles in behavioral variant frontotemporal dementia. Biol Psychiatry, 75(7), 582-588. doi: 10.1016/j.biopsych.2013.08.017

Butts, A. M., Machulda, M. M., Duffy, J. R., Strand, E. A., Whitwell, J. L., \& Josephs, K. A. (2015). Neuropsychological Profiles Differ among the Three Variants of Primary Progressive Aphasia. J Int Neuropsychol Soc, 21(6), 429435. doi: $10.1017 / \mathrm{S} 1355617715000399$

Casaletto, K. B., Marx, G., Dutt, S., Neuhaus, J., Saloner, R., Kritikos, L., . . . Kramer, J. H. (2017). Is "Learning" episodic memory? Distinct cognitive and neuroanatomic correlates of immediate recall during learning trials in neurologically normal aging and neurodegenerative cohorts.

Neuropsychologia, 102, 19-28. doi: 10.1016/j.neuropsychologia.2017.05.021

Caspers, S., Eickhoff, S. B., Rick, T., von Kapri, A., Kuhlen, T., Huang, R., . . Zilles, K. (2011). Probabilistic fibre tract analysis of cytoarchitectonically defined human inferior parietal lobule areas reveals similarities to macaques. Neuroimage, 58(2), 362-380. doi: 10.1016/j.neuroimage.2011.06.027

Catani, M., Howard, R. J., Pajevic, S., \& Jones, D. K. (2002). Virtual in vivo interactive dissection of white matter fasciculi in the human brain. Neuroimage, 17(1), 77-94.

Chare, L., Hodges, J. R., Leyton, C. E., McGinley, C., Tan, R. H., Kril, J. J., \& Halliday, G. M. (2014). New criteria for frontotemporal dementia syndromes: clinical and pathological diagnostic implications. J Neurol Neurosurg Psychiatry, 85(8), 865-870. doi: 10.1136/jnnp-2013-306948

Desgranges, B., Baron, J. C., Lalevee, C., Giffard, B., Viader, F., de La Sayette, V., \& Eustache, F. (2002). The neural substrates of episodic memory impairment in Alzheimer's disease as revealed by FDG-PET: relationship to degree of deterioration. Brain, 125(Pt 5), 1116-1124. 
Dickerson, B. C., \& Eichenbaum, H. (2010). The episodic memory system: neurocircuitry and disorders. Neuropsychopharmacology, 35(1), 86-104. doi: 10.1038/npp.2009.126

Eikelboom, W. S., Janssen, N., Jiskoot, L. C., van den Berg, E., Roelofs, A., \& Kessels, R. P. C. (2018). Episodic and working memory function in Primary Progressive Aphasia: A meta-analysis. Neurosci Biobehav Rev. doi: 10.1016/j.neubiorev.2018.06.015

Galantucci, S., Tartaglia, M. C., Wilson, S. M., Henry, M. L., Filippi, M., Agosta, F., . . Gorno-Tempini, M. L. (2011). White matter damage in primary progressive aphasias: a diffusion tensor tractography study. Brain, 134, 30113029. doi: 10.1093/brain/awr099

Gilmore, A. W., Nelson, S. M., \& McDermott, K. B. (2015). A parietal memory network revealed by multiple MRI methods. Trends Cogn Sci, 19(9), 534-543. doi: 10.1016/j.tics.2015.07.004

Gorno-Tempini, M. L., Brambati, S. M., Ginex, V., Ogar, J., Dronkers, N. F., Marcone, A., . . Miller, B. L. (2008). The logopenic/phonological variant of primary progressive aphasia. Neurology, 71(16), 1227-1234. doi: 10.1212/01.wnl.0000320506.79811.da

Gorno-Tempini, M. L., Dronkers, N. F., Rankin, K. P., Ogar, J. M., Phengrasamy, L., Rosen, H. J., . . Miller, B. L. (2004). Cognition and anatomy in three variants of primary progressive aphasia. Ann Neurol, 55(3), 335-346. doi: 10.1002/ana. 10825

Gorno-Tempini, M. L., Hillis, A. E., Weintraub, S., Kertesz, A., Mendez, M., Cappa, S. F., . . Grossman, M. (2011). Classification of primary progressive aphasia and its variants. Neurology, 76(11), 1006-1014. doi: 10.1212/WNL.0b013e31821103e6

Gow, D. W., Jr. (2012). The cortical organization of lexical knowledge: a dual lexicon model of spoken language processing. Brain Lang, 121(3), 273-288. doi: 10.1016/j.bandl.2012.03.005

Graham, N. L., Emery, T., \& Hodges, J. R. (2004). Distinctive cognitive profiles in Alzheimer's disease and subcortical vascular dementia. J Neurol Neurosurg Psychiatry, 75(1), 61-71.

Grossman, M. (2010). Primary progressive aphasia: clinicopathological correlations. Nat Rev Neurol, 6(2), 88-97. doi: 10.1038/nrneurol.2009.216

Hagmann, P., Cammoun, L., Gigandet, X., Meuli, R., Honey, C. J., Wedeen, V. J., \& Sporns, O. (2008). Mapping the structural core of human cerebral cortex. PLoS Biol, 6(7), e159. doi: 10.1371/journal.pbio.0060159

Henry, M. L., \& Gorno-Tempini, M. L. (2010). The logopenic variant of primary progressive aphasia. Curr Opin Neurol, 23(6), 633-637. doi: 10.1097/WCO.0b013e32833fb93e

Herbet, G., Zemmoura, I., \& Duffau, H. (2018). Functional Anatomy of the Inferior Longitudinal Fasciculus: From Historical Reports to Current Hypotheses. [Review]. Frontiers in Neuroanatomy, 12(77). doi: 10.3389/fnana.2018.00077

Hua, K., Zhang, J., Wakana, S., Jiang, H., Li, X., Reich, D. S., . . Mori, S. (2008). Tract probability maps in stereotaxic spaces: analyses of white matter anatomy and tract-specific quantification. Neuroimage, 39(1), 336-347. doi: 10.1016/j.neuroimage.2007.07.053

Hutchinson, A. D., \& Mathias, J. L. (2007). Neuropsychological deficits in frontotemporal dementia and Alzheimer's disease: a meta-analytic review. $J$ Neurol Neurosurg Psychiatry, 78(9), 917-928. doi: 10.1136/jnnp.2006.100669 
Irish, M., Addis, D. R., Hodges, J. R., \& Piguet, O. (2012). Considering the role of semantic memory in episodic future thinking: evidence from semantic dementia. Brain, 135(Pt 7), 2178-2191. doi: 10.1093/brain/aws119

Irish, M., Bunk, S., Tu, S., Kamminga, J., Hodges, J. R., Hornberger, M., \& Piguet, O. (2016). Preservation of episodic memory in semantic dementia: The importance of regions beyond the medial temporal lobes. Neuropsychologia, 81, 50-60. doi: 10.1016/j.neuropsychologia.2015.12.005

Irish, M., Halena, S., Kamminga, J., Tu, S., Hornberger, M., \& Hodges, J. R. (2015). Scene construction impairments in Alzheimer's disease - A unique role for the posterior cingulate cortex. Cortex, 73, 10-23. doi: 10.1016/j.cortex.2015.08.004

Irish, M., Piguet, O., Hodges, J. R., \& Hornberger, M. (2014). Common and unique gray matter correlates of episodic memory dysfunction in frontotemporal dementia and Alzheimer's disease. Hum Brain Mapp, 35(4), 1422-1435. doi: $10.1002 / \mathrm{hbm} .22263$

Karas, G. B., Scheltens, P., Rombouts, S. A., Visser, P. J., van Schijndel, R. A., Fox, N. C., \& Barkhof, F. (2004). Global and local gray matter loss in mild cognitive impairment and Alzheimer's disease. Neuroimage, 23(2), 708-716. doi: S1053-8119(04)00371-4 [pii]

10.1016/j.neuroimage.2004.07.006 [doi]

Kelley, W. M., Miezin, F. M., McDermott, K. B., Buckner, R. L., Raichle, M. E., Cohen, N. J., . . Petersen, S. E. (1998). Hemispheric specialization in human dorsal frontal cortex and medial temporal lobe for verbal and nonverbal memory encoding. Neuron, 20(5), 927-936.

Khan, U. A., Liu, L., Provenzano, F. A., Berman, D. E., Profaci, C. P., Sloan, R., .. . Small, S. A. (2014). Molecular drivers and cortical spread of lateral entorhinal cortex dysfunction in preclinical Alzheimer's disease. Nat Neurosci, 17(2), 304-311. doi: 10.1038/nn.3606

Knopman, D. S., Kramer, J. H., Boeve, B. F., Caselli, R. J., Graff-Radford, N. R., Mendez, M. F., . . Mercaldo, N. (2008). Development of methodology for conducting clinical trials in frontotemporal lobar degeneration. Brain, 131(Pt 11), 2957-2968. doi: 10.1093/brain/awn234

Krishnan, K., Machulda, M. M., Whitwell, J. L., Butts, A. M., Duffy, J. R., Strand, E. A., . . Josephs, K. A. (2017). Varying Degrees of Temporoparietal Hypometabolism on FDG-PET Reveal Amyloid-Positive Logopenic Primary Progressive Aphasia is not a Homogeneous Clinical Entity. J Alzheimers Dis, 55(3), 1019-1029. doi: 10.3233/JAD-160614

Leyton, C. E., Hodges, J. R., McLean, C. A., Kril, J. J., Piguet, O., \& Ballard, K. J. (2015). Is the logopenic-variant of primary progressive aphasia a unitary disorder? Cortex, 67, 122-133. doi: 10.1016/j.cortex.2015.03.011

Leyton, C. E., Piguet, O., Savage, S., Burrell, J., \& Hodges, J. R. (2012). The neural basis of logopenic progressive aphasia. J Alzheimers Dis, 32(4), 1051-1059. doi: 10.3233/JAD-2012-121042

Leyton, C. E., Villemagne, V. L., Savage, S., Pike, K. E., Ballard, K. J., Piguet, O., . . . Hodges, J. R. (2011). Subtypes of progressive aphasia: application of the International Consensus Criteria and validation using beta-amyloid imaging. Brain, 134(Pt 10), 3030-3043. doi: 10.1093/brain/awr216

Magnin, E., Chopard, G., Ferreira, S., Sylvestre, G., Dariel, E., Ryff, I., . . Rumbach, L. (2013). Initial neuropsychological profile of a series of 20 patients with 
logopenic variant of primary progressive aphasia. J Alzheimers Dis, 36(4), 799-808. doi: 10.3233/JAD-122335

McKhann, G. M., Knopman, D. S., Chertkow, H., Hyman, B. T., Jack, C. R., Jr., Kawas, C. H., . . . Phelps, C. H. (2011). The diagnosis of dementia due to Alzheimer's disease: recommendations from the National Institute on AgingAlzheimer's Association workgroups on diagnostic guidelines for Alzheimer's disease. Alzheimers Dement, 7(3), 263-269. doi: 10.1016/j.jalz.2011.03.005

Mioshi, E., Dawson, K., Mitchell, J., Arnold, R., \& Hodges, J. R. (2006). The Addenbrooke's Cognitive Examination Revised (ACE-R): a brief cognitive test battery for dementia screening. Int J Geriatr Psychiatry, 21(11), 10781085. doi: 10.1002/gps.1610

Moller, C., Vrenken, H., Jiskoot, L., Versteeg, A., Barkhof, F., Scheltens, P., \& van der Flier, W. M. (2013). Different patterns of gray matter atrophy in early- and late-onset Alzheimer's disease. Neurobiol Aging, 34(8), 2014-2022. doi: 10.1016/j.neurobiolaging.2013.02.013

Nadel, L., \& Moscovitch, M. (1997). Memory consolidation, retrograde amnesia and the hippocampal complex. Curr Opin Neurobiol, 7(2), 217-227.

Osterrieth, P. (1944). Filetest de copie d'une figure complex: Contribution a l'etude de la perception et de la memoire [The test of copying a complex figure: A contribution to the study of perception and memory]. Archives de Psychologie, 30, 286-356.

Piguet, O., Leyton, C. E., Gleeson, L. D., Hoon, C., \& Hodges, J. R. (2015). Memory and emotion processing performance contributes to the diagnosis of nonsemantic primary progressive aphasia syndromes. J Alzheimers Dis, 44(2), 541-547. doi: 10.3233/JAD-141854

Ramanan, S., \& Bellana, B. (2019). A Domain-General Role for the Angular Gyrus in Retrieving Internal Representations of the External World. J Neurosci, 39(16), 2978-2980. doi: 10.1523/JNEUROSCI.3231-18.2019

Ramanan, S., Bertoux, M., Flanagan, E., Irish, M., Piguet, O., Hodges, J. R., \& Hornberger, M. (2017). Longitudinal Executive Function and Episodic Memory Profiles in Behavioral-Variant Frontotemporal Dementia and Alzheimer's Disease. J Int Neuropsychol Soc, 23(1), 34-43. doi: $10.1017 / \mathrm{S} 1355617716000837$

Ramanan, S., Flanagan, E., Leyton, C. E., Villemagne, V. L., Rowe, C. C., Hodges, J. R., \& Hornberger, M. (2016). Non-Verbal Episodic Memory Deficits in Primary Progressive Aphasias are Highly Predictive of Underlying Amyloid Pathology. J Alzheimers Dis, 51(2), 367-376. doi: 10.3233/JAD-150752

Ramanan, S., Piguet, O., \& Irish, M. (2018). Rethinking the Role of the Angular Gyrus in Remembering the Past and Imagining the Future: The Contextual Integration Model. Neuroscientist, 24(4), 342-352. doi: $10.1177 / 1073858417735514$

Ramanan, S., Strikwerda-Brown, C., Mothakunnel, A., Hodges, J. R., Piguet, O., \& Irish, M. (2019). Fronto-parietal contributions to episodic retrieval-evidence from neurodegenerative disorders. Learn Mem, 26(7), 262-271. doi: $10.1101 / \mathrm{lm} .048454 .118$

Reitan, R. M. (1958). Validity of the Trail Making Test as an indicator of organic brain damage. Perceptual and Motor Skills, 8, 271-276.

Rogalski, E., Cobia, D., Martersteck, A., Rademaker, A., Wieneke, C., Weintraub, S., \& Mesulam, M. M. (2014). Asymmetry of cortical decline in subtypes of 
primary progressive aphasia. Neurology, 83(13), 1184-1191. doi: 10.1212/WNL.0000000000000824

Rohrer, J. D., Caso, F., Mahoney, C., Henry, M., Rosen, H. J., Rabinovici, G., . . . Gorno-Tempini, M. L. (2013). Patterns of longitudinal brain atrophy in the logopenic variant of primary progressive aphasia. Brain Lang, 127(2), 121126. doi: 10.1016/j.band1.2012.12.008

Rohrer, J. D., Ridgway, G. R., Crutch, S. J., Hailstone, J., Goll, J. C., Clarkson, M. J., . . Warren, J. D. (2010). Progressive logopenic/phonological aphasia: erosion of the language network. Neuroimage, 49(1), 984-993. doi: 10.1016/j.neuroimage.2009.08.002

Rohrer, J. D., Rossor, M. N., \& Warren, J. D. (2012). Alzheimer's pathology in primary progressive aphasia. Neurobiol Aging, 33(4), 744-752. doi: 10.1016/j.neurobiolaging.2010.05.020

Rushworth, M. F., Behrens, T. E., \& Johansen-Berg, H. (2006). Connection patterns distinguish 3 regions of human parietal cortex. Cereb Cortex, 16(10), 14181430. doi: 10.1093/cercor/bhj079

Savage, S., Hsieh, S., Leslie, F., Foxe, D., Piguet, O., \& Hodges, J. R. (2013). Distinguishing subtypes in primary progressive aphasia: application of the Sydney language battery. Dement Geriatr Cogn Disord, 35(3-4), 208-218. doi: $10.1159 / 000346389$

Schmidt, M. (1996). Rey auditory verbal learning test: A handbook. Los Angeles, USA: Western Psychological Services.

Smith, S. M. (2002). Fast robust automated brain extraction. Hum Brain Mapp, 17(3), 143-155. doi: 10.1002/hbm.10062 [doi]

Smith, S. M., Jenkinson, M., Johansen-Berg, H., Rueckert, D., Nichols, T. E., Mackay, C. E., .. . Behrens, T. E. (2006). Tract-based spatial statistics: voxelwise analysis of multi-subject diffusion data. Neuroimage, 31(4), 14871505. doi: 10.1016/j.neuroimage.2006.02.024

Strauss, E., Sherman, E. M. S., \& Spreen, O. (2006). A Compendium of Neuropsychological Tests: Administration, Norms, and Commentary (3rd Edition ed.): Oxford University Press.

Teichmann, M., Kas, A., Boutet, C., Ferrieux, S., Nogues, M., Samri, D., . . . Migliaccio, R. (2013). Deciphering logopenic primary progressive aphasia: a clinical, imaging and biomarker investigation. Brain, 136(Pt 11), 3474-3488. doi: 10.1093/brain/awt266

Tu, S., Leyton, C. E., Hodges, J. R., Piguet, O., \& Hornberger, M. (2016). Divergent Longitudinal Propagation of White Matter Degradation in Logopenic and Semantic Variants of Primary Progressive Aphasia. J Alzheimers Dis, 49(3), 853-861. doi: 10.3233/JAD-150626

Uddin, L. Q., Supekar, K., Amin, H., Rykhlevskaia, E., Nguyen, D. A., Greicius, M. D., \& Menon, V. (2010). Dissociable connectivity within human angular gyrus and intraparietal sulcus: evidence from functional and structural connectivity. Cereb Cortex, 20(11), 2636-2646. doi: 10.1093/cercor/bhq011

Villain, N., Desgranges, B., Viader, F., de la Sayette, V., Mezenge, F., Landeau, B., . . . Chetelat, G. (2008). Relationships between hippocampal atrophy, white matter disruption, and gray matter hypometabolism in Alzheimer's disease. Journal of Neuroscience, 28(24), 6174-6181. doi: 10.1523/Jneurosci.139208.2008

Vincent, J. L., Snyder, A. Z., Fox, M. D., Shannon, B. J., Andrews, J. R., Raichle, M. E., \& Buckner, R. L. (2006). Coherent spontaneous activity identifies a 
hippocampal-parietal memory network. J Neurophysiol, 96(6), 3517-3531. doi: 10.1152/jn.00048.2006

Wear, H. J., Wedderburn, C. J., Mioshi, E., Williams-Gray, C. H., Mason, S. L., Barker, R. A., \& Hodges, J. R. (2008). The Cambridge Behavioural Inventory revised. Dement Neuropsychol, 2(2), 102-107.

Wechsler, D. (1997). Wechsler Adult Intelligence Scale - 3rd Edition (WAIS-3®). San Antonio, TX: Harcourt Assessment.

Win, K. T., Pluta, J., Yushkevich, P., Irwin, D. J., McMillan, C. T., Rascovsky, K., . . . Grossman, M. (2017). Neural Correlates of Verbal Episodic Memory and Lexical Retrieval in Logopenic Variant Primary Progressive Aphasia. Front Neurosci, 11, 330. doi: 10.3389/fnins.2017.00330

Zhang, Y. Y., Brady, M., \& Smith, S. (2001). Segmentation of brain MR images through a hidden Markov random field model and the expectationmaximization algorithm. Ieee Transactions on Medical Imaging, 20(1), 45-57. doi: Doi 10.1109/42.906424 


\section{Author contributions}

Siddharth Ramanan: Conceptualization; Methodology; Validation; Formal analysis; Investigation; Visualization; Writing - Original draft; Writing - Review \& Editing.

Lars Marstaller: Methodology; Software; Validation; Visualization; Writing - Review \& Editing.

John R. Hodges: Resources; Writing - Review \& Editing.

Olivier Piguet: Investigation; Resources; Visualization; Writing - Review \& Editing; Supervision

Muireann Irish: Conceptualization; Methodology; Validation; Formal analysis; Investigation; Resources; Visualization; Writing - Original draft; Writing - Review \& Editing; Supervision. 

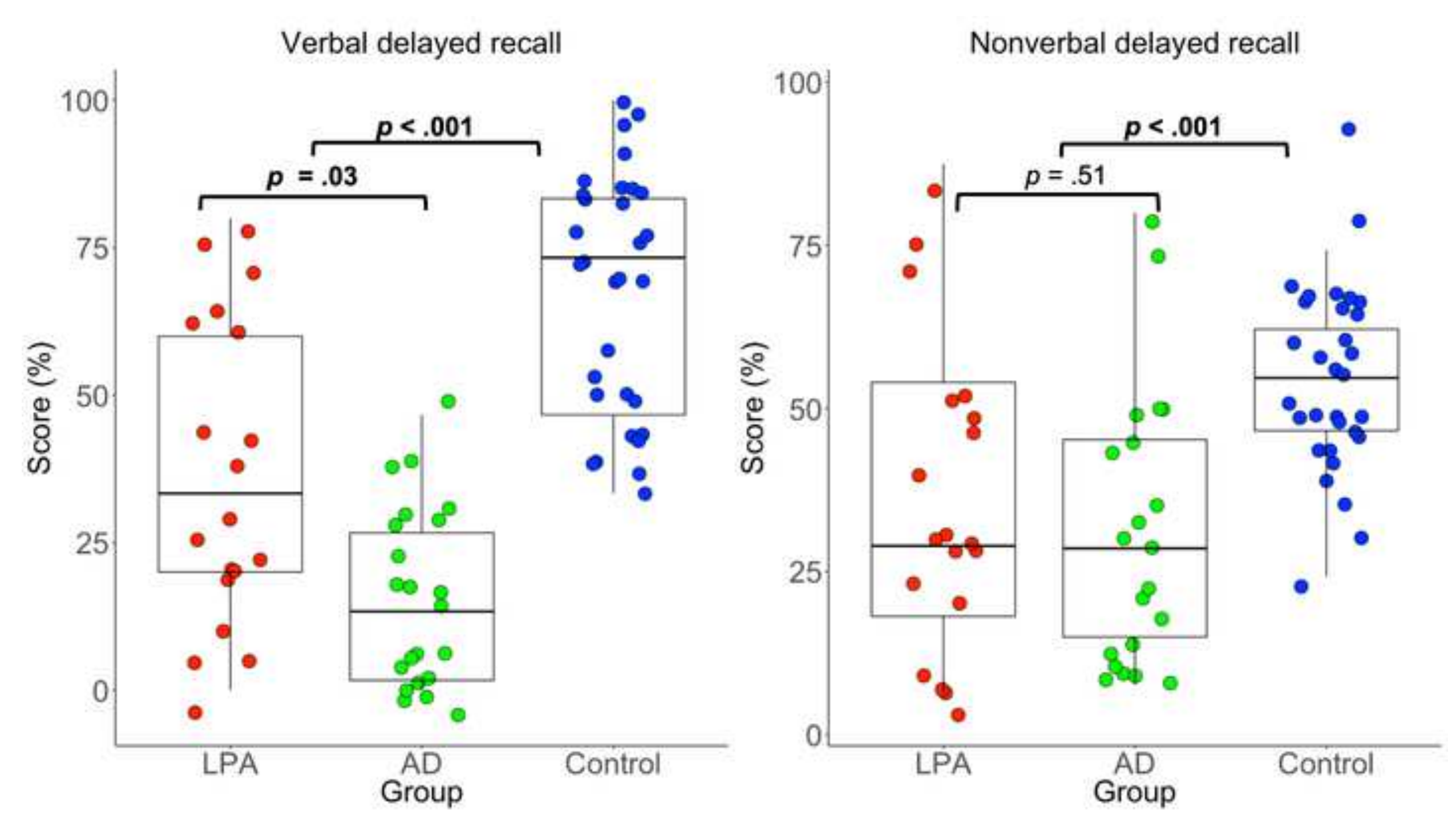


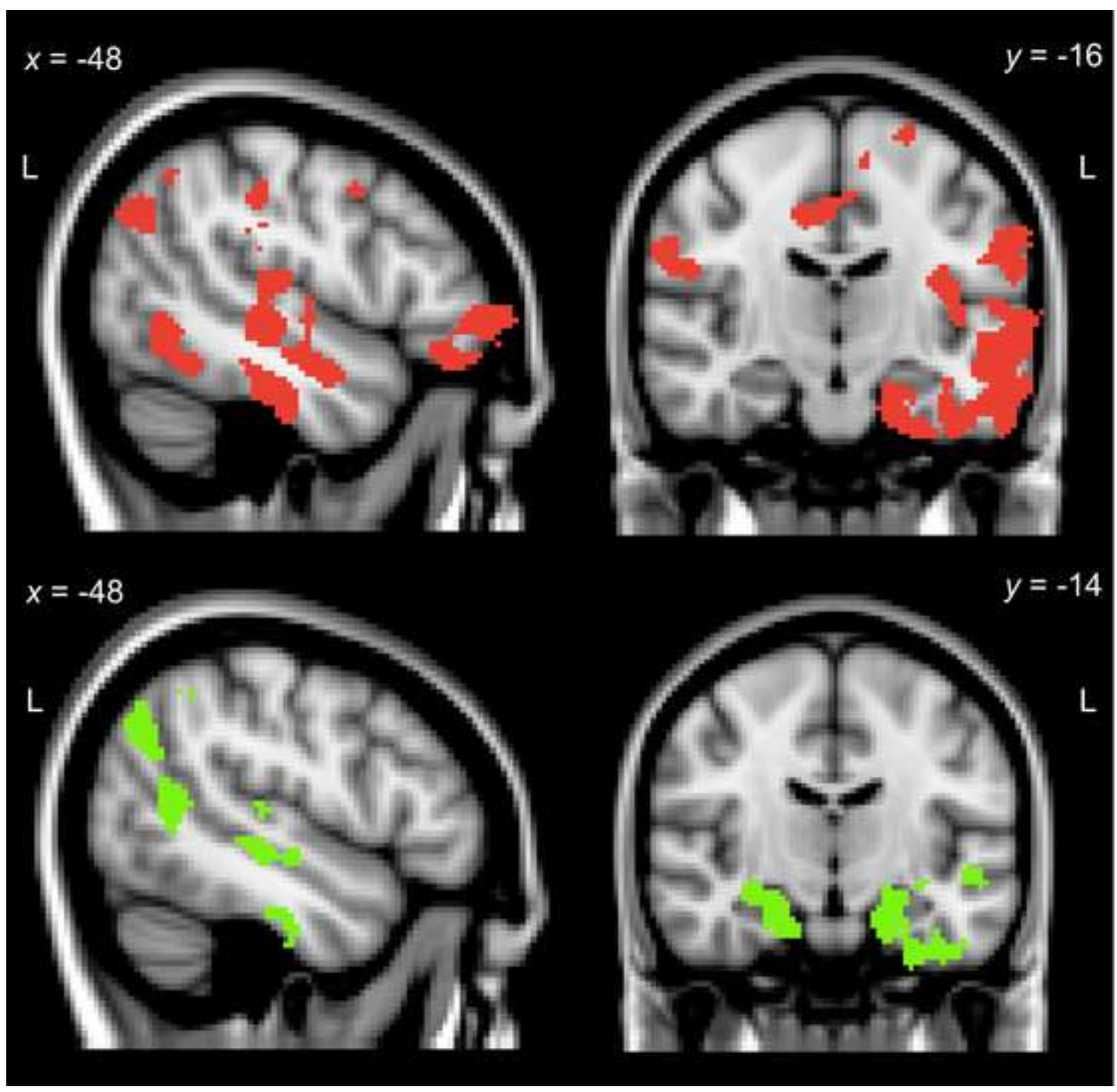

Group

- LPA

- $A D$

L 


$$
x=-40 \quad x=-10
$$
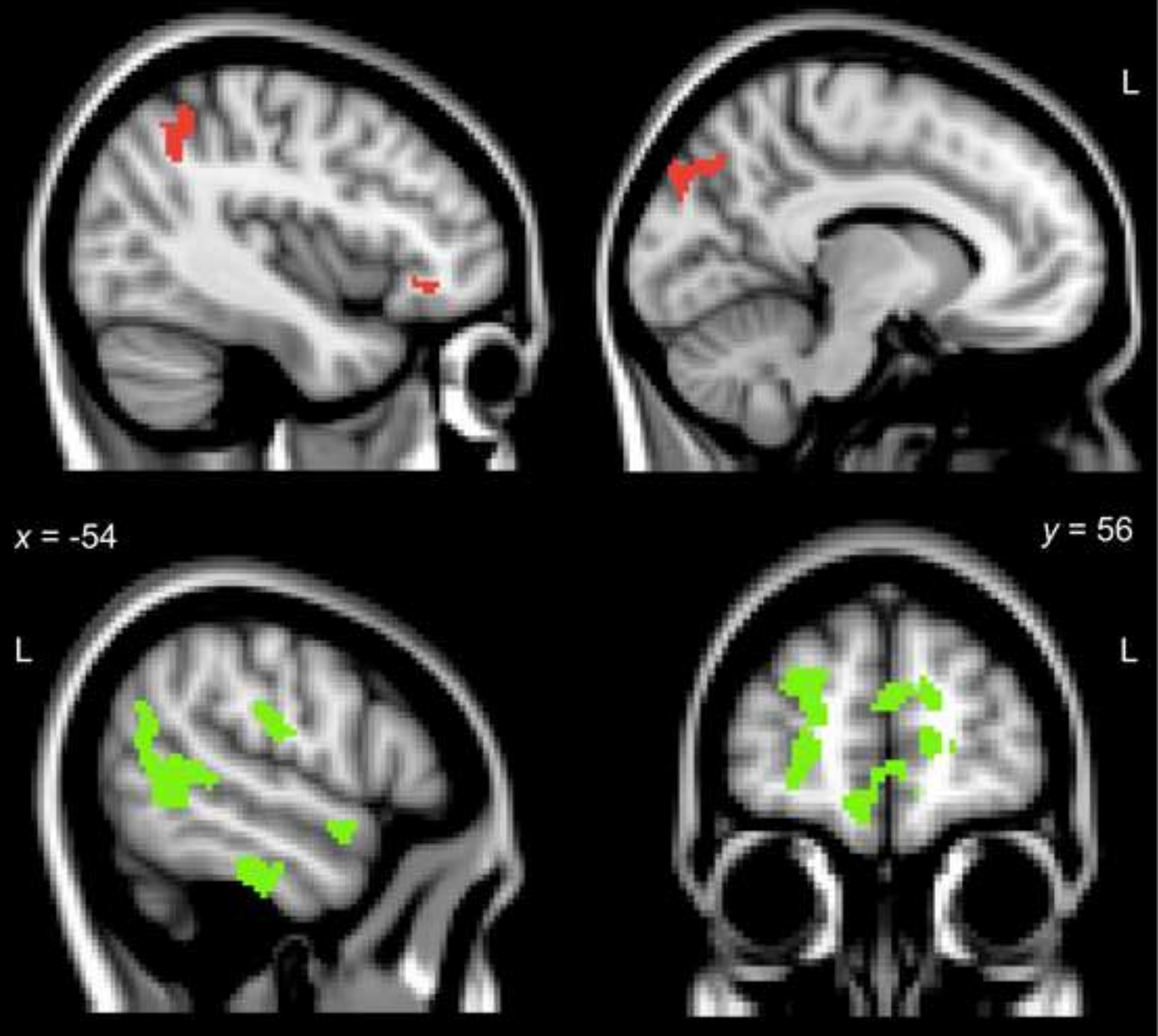

Group - LPA

- $A D$ 


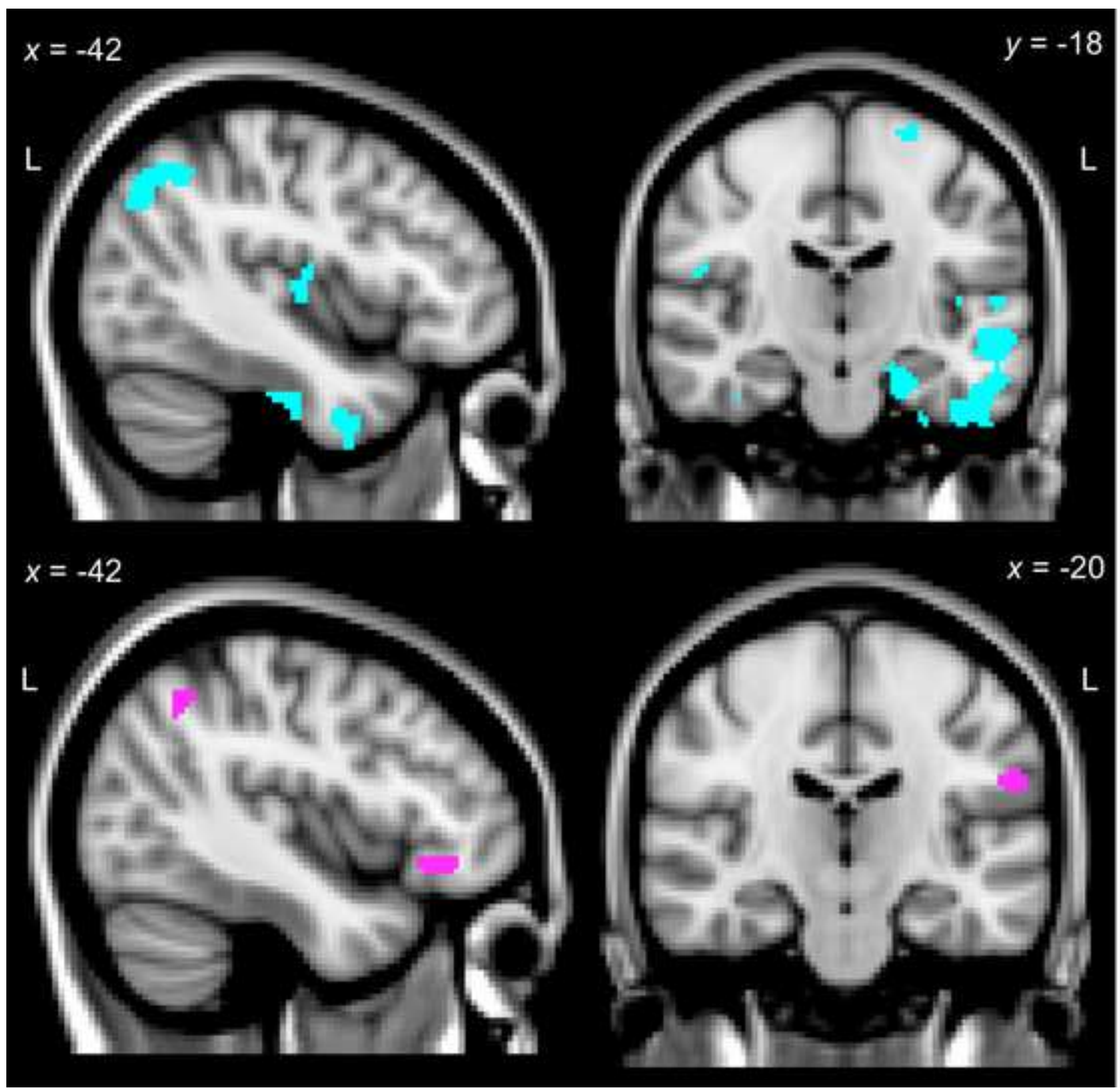

Measure

Verbal delayed recall

- Nonverbal delayed recall 


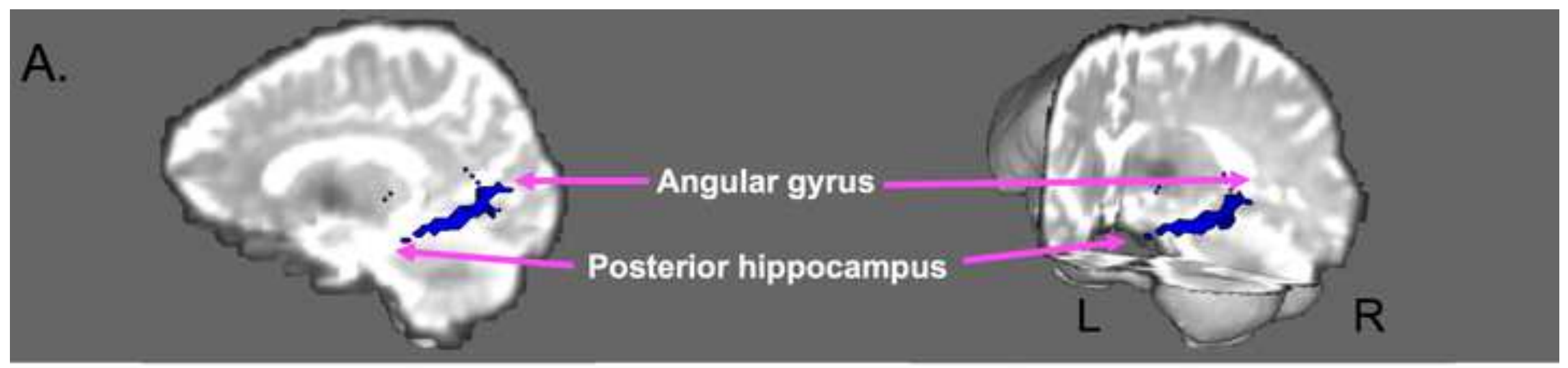

B. Mean diffusivity

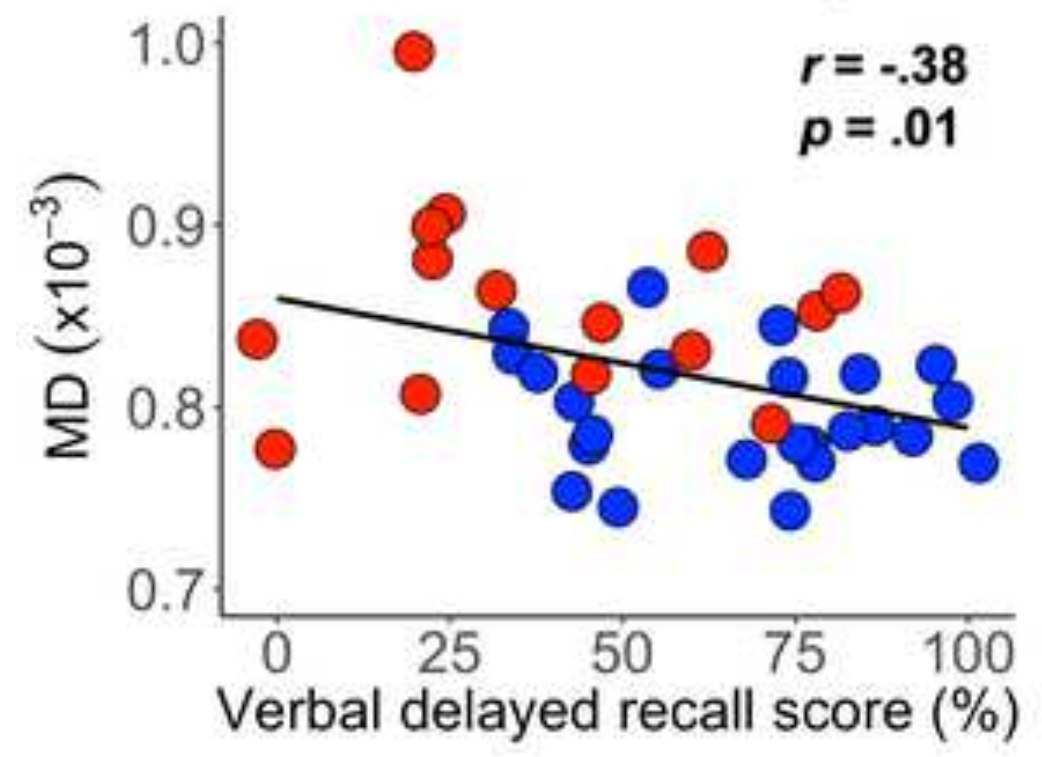

C. Mean diffusivity

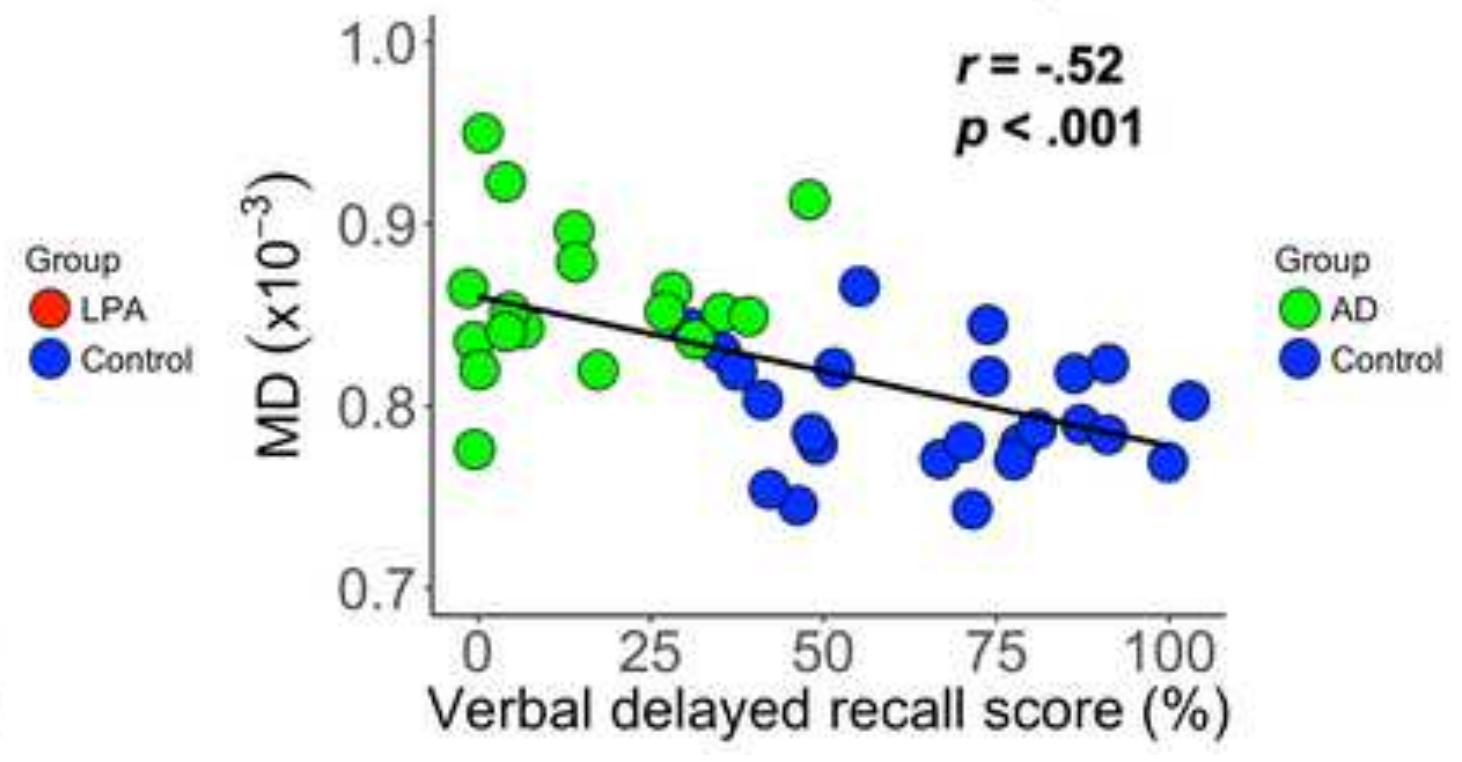

